\title{
Article
}

\section{High-Frequency Instabilities of a Boussinesq-Whitham System: A Perturbative Approach}

\author{
Ryan Creedon ${ }^{1, *}$, Bernard Deconinck ${ }^{1}$ and Olga Trichtchenko ${ }^{2}$ \\ 1 Department of Applied Mathematics, University of Washington, Seattle, WA 98195, USA; deconinc@uw.edu \\ 2 Department of Physics and Astronomy, The University of Western Ontario, London, ON N6A 3K7, Canada; \\ otrichtc@uwo.ca \\ * Correspondence: creedon@uw.edu
}

check for updates

Citation: Creedon, R.; Deconinck, B.; Trichtchenko, O. High-Frequency Instabilities of a Boussinesq-Whitham System: A Perturbative Approach. Fluids 2021, 6, 136. https://doi.org/ 10.3390 /fluids6040136

Academic Editor: Alexander I. Dyachenko

Received: 9 February 2021

Accepted: 26 March 2021

Published: 1 April 2021

Publisher's Note: MDPI stays neutral with regard to jurisdictional claims in published maps and institutional affiliations.

Copyright: (c) 2021 by the authors. Licensee MDPI, Basel, Switzerland. This article is an open access article distributed under the terms and conditions of the Creative Commons Attribution (CC BY) license (https:// creativecommons.org/licenses/by/ $4.0 /)$.

\begin{abstract}
We analyze the spectral stability of small-amplitude, periodic, traveling-wave solutions of a Boussinesq-Whitham system. These solutions are shown numerically to exhibit high-frequency instabilities when subject to bounded perturbations on the real line. We use a formal perturbation method to estimate the asymptotic behavior of these instabilities in the small-amplitude regime. We compare these asymptotic results with direct numerical computations.
\end{abstract}

Keywords: Boussinesq-Whitham systems; traveling waves; spectral stability; high-frequency instabilities; perturbation methods

\section{Introduction}

In 1967, Whitham [1] proposed a nonlinear, integro-differential equation to model shallow water waves with a linearized dispersion relation that matches one full branch of the 1D Euler water wave problem (WWP) [2]. Because its dispersion relation excludes the other branch, however, Whitham's equation does not capture all the instabilities of traveling-wave solutions of the WWP [3].

In this manuscript, we investigate the spectral stability of small-amplitude, $2 \pi / \kappa-$ periodic, traveling waves of a Boussinesq-Whitham system proposed by Hur and Pandey [4] and Hur and Tao [5]:

$$
\begin{aligned}
& \eta_{t}=-h_{0} u_{x}-(\eta u)_{x}, \\
& u_{t}=-g \mathcal{K}\left[\eta_{x}\right]-u u_{x} .
\end{aligned}
$$

In this model, $\eta(x, t)$ represents the displacement of a wave profile from its equilibrium depth $h_{0}, u(x, t)$ is the horizontal velocity along $\eta$, and $\mathcal{K}$ is a Fourier multiplier operator defined so that the linearized dispersion relation of (1) matches both branches of the WWP. For functions $f \in \mathrm{L}_{\text {per }}^{1}(-\pi / \kappa, \pi / \kappa), \mathcal{K}$ is defined as

$$
\widehat{\mathcal{K}[f]}(\kappa n)=\frac{\tanh \left(\kappa n h_{0}\right)}{\kappa n h_{0}} \widehat{f}(\kappa n), \quad n \in \mathbb{Z},
$$

where $\widehat{\cdot}$ denotes the Fourier transform of $f$ :

$$
\widehat{f}(k)=\frac{\kappa}{2 \pi} \int_{-\pi / \kappa}^{\pi / \kappa} f(x) e^{-i k x} d x .
$$

Alternatively, $\mathcal{K}$ can be defined in physical variables as the pseudo-differential operator

$$
\mathcal{K}[f]=\left(\frac{\tanh \left(h_{0} D\right)}{h_{0} D}\right) f \text {, }
$$

where $D=-i \partial_{x}$. For the remainder of this manuscript, we refer to (1) as the Hur-PandeyTao-Boussinesq-Whitham system, or HPT-BW for short. 
The HPT-BW system is Hamiltonian [4], with

$$
\mathcal{H}=\frac{1}{2} \int_{-\pi / \kappa}^{\pi / \kappa}\left(h_{0} u^{2}+g \eta \mathcal{K}[\eta]+\eta u^{2}\right) d x,
$$

and non-canonical Poisson structure

$$
J=-\left(\begin{array}{cc}
0 & \partial_{x} \\
\partial_{x} & 0
\end{array}\right)
$$

The system has a one-parameter family of small-amplitude, $2 \pi / \kappa$-periodic, travelingwave solutions for each $\kappa>0$. We call these solutions the Stokes waves of HPT-BW by analogy with solutions of the WWP of the same name [6-8]. In Section 2, we derive a power series expansion for HPT-BW Stokes waves in a small parameter $\varepsilon$ that scales with the amplitude of the waves.

Perturbing Stokes waves yield a spectral problem after linearizing the governing equations of the perturbations. The spectral elements of this problem define the stability spectrum of Stokes waves (Section 3). The stability spectrum is purely continuous [9-11], but Floquet theory decomposes the spectrum into an uncountable union of point spectra. Each of these point spectra is indexed by the Floquet exponent [12-14].

The stability spectrum inherits quadrafold symmetry from the Hamiltonian structure of (1); i.e., the spectrum is invariant under conjugation and negation $[10,13]$. Because of quadrafold symmetry, all elements of the stability spectrum have non-positive real component only if the stability spectrum is a subset of the imaginary axis. Therefore, HPT-BW Stokes waves are spectrally stable only if the spectrum lies on the imaginary axis. Otherwise, the Stokes waves are spectrally unstable.

If the aspect ratio $\kappa h_{0}$ is sufficiently large, both HPT-BW [4] and WWP Stokes waves [15-17] have stability spectra near the origin that leave the imaginary axis for $0<|\varepsilon| \ll 1$, resulting in modulational instability. Using the Floquet-Fourier-Hill (FFH) method [12], recent numerical work by [18] and [19,20] shows, respectively, that HPT-BW and WWP Stokes waves also have stability spectra away from the origin that leave the imaginary axis, regardless of $k h_{0}$. These spectra give rise to the so-called high-frequency instabilities [3], shown schematically in Figure 1.
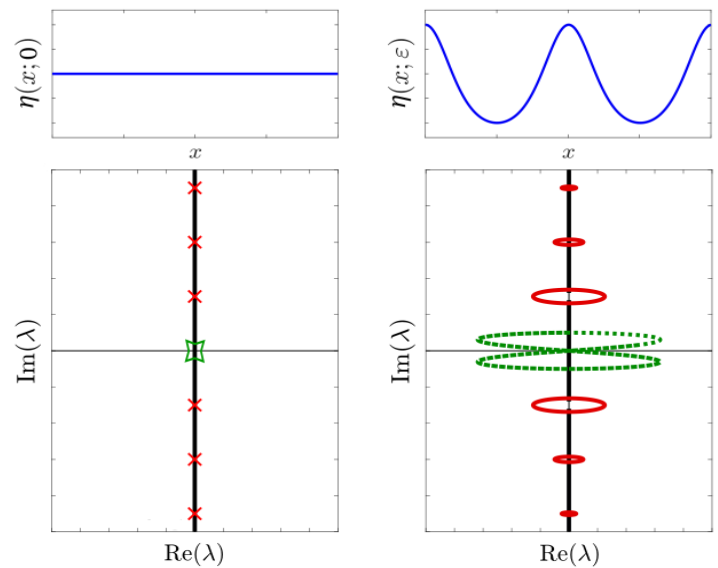

Figure 1. (Top, left) A zero-amplitude $(\varepsilon=0)$ Stokes wave (solid blue). (Bottom, left) The stability spectrum of the zero-amplitude Stokes wave (solid black). Collisions of nonzero spectral elements are denoted by red crosses, while the collision of spectral elements at the origin is denoted by the green polygon. (Top, right) A small-amplitude $(0<|\varepsilon| \ll 1)$ Stokes wave (solid blue). (Bottom, right) The stability spectrum of the small-amplitude Stokes wave. Stable elements are in solid black. High-frequency isolas are in solid red. The modulational instability is in dashed green and present only if $\kappa h_{0}$ is sufficiently large. 
High-frequency instabilities arise from the collision of nonzero elements of the zeroamplitude $(\varepsilon=0)$ stability spectrum. At these collided spectral elements, a HamiltonianHopf bifurcation occurs, resulting in a locus of spectral elements bounded away from the origin that leave the imaginary axis as $|\varepsilon|$ increases. We refer to this locus of spectral elements as a high-frequency isola.

High-frequency isolas are difficult to find using numerical methods like FFH. To capture the isola closest to the origin, for example, the corresponding interval of Floquet exponents has width $\mathcal{O}\left(\varepsilon^{2}\right)$ (Figure 2). In general, the $j$ th isola from the origin appears to have width $O\left(\varepsilon^{j+1}\right)$. The isolas also drift from their initial collision sites (Figure 2), meaning that the Floquet exponent that gives rise to the collided spectral elements at $\varepsilon=0$ is not contained in the interval parameterizing the corresponding isola for sufficiently large $|\varepsilon|$.To circumvent these difficulties, one must supply the numerical method with asymptotic expressions for the interval of Floquet exponents corresponding to the desired isolas. We discover these expressions for high-frequency isolas of HPT-BW.
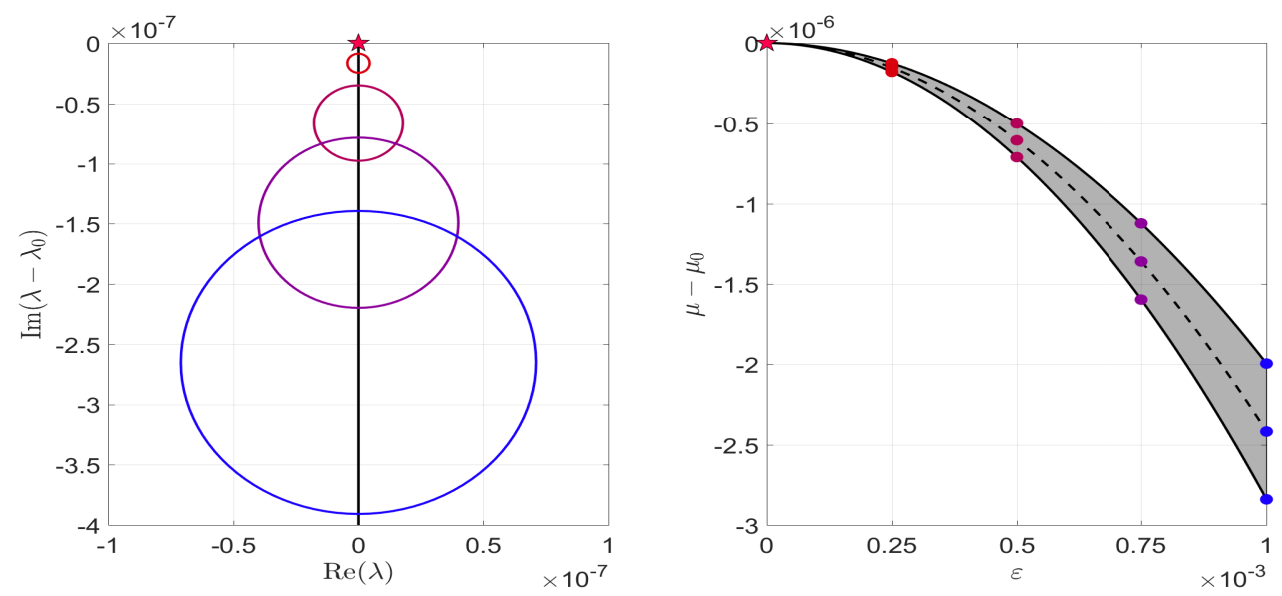

Figure 2. (Left) The high-frequency isola of HPT-BW closest to the origin with $\kappa=g=h_{0}=1$ and $\varepsilon=2.5 \times 10^{-4}$ (red), $5 \times 10^{-4}$ (burgundy), $7.5 \times 10^{-4}$ (purple), and $10^{-3}$ (blue). The collision that generates the isola when $\varepsilon=0$ is indicated by the red star. The imaginary axis is recentered to show the magnitude of the drift of the isola from the collision site $\lambda_{0}$. (Right) Interval of Floquet exponents that parameterize the isola as a function of $\varepsilon$. The solid black lines indicate the boundaries of the interval, while the dashed black line gives the Floquet exponent of the most unstable spectral element of the isola. The colored dots provide the Floquet data for the correspondingly colored isola in the left plot. The red star indicates the Floquet exponent $\mu_{0}$ that generates the isola when $\varepsilon=0$. The Floquet axis is recentered to show the magnitude of the drift of the Floquet exponents from $\mu_{0}$.

Our motivation for studying the HPT-BW system, apart from its inherent interest, is that it retains the full dispersion relation (both branches) of the more complicated WWP. Our goal is the application of the perturbation method developed herein to the WWP. The first step towards this goal was the investigation of the stability spectra of Stokes waves of the Kawahara Equation [21]. The investigations presented here constitute our second step, before we proceed to the WWP.

For a given high-frequency isola of an HPT-BW Stokes wave, we obtain (i) an asymptotic range of Floquet exponents that parameterizes the isola, (ii) an asymptotic estimate for the most unstable spectral element of the isola, (iii) expressions of curves that are asymptotic to the isola, and (iv) wavenumbers for which the given isola is not present. Our approach is inspired by a perturbation method outlined in [22], but modified appropriately for higher-order calculations. We compare all asymptotic results with numerical results computed by the FFH method. 


\section{Small-Amplitude Stokes Waves}

In a traveling frame moving with velocity $c, x \rightarrow x-c t$ and (1) becomes

$$
\begin{aligned}
& \eta_{t}=c \eta_{x}-h_{0} u_{x}-(\eta u)_{x} \\
& u_{t}=c u_{x}-g \mathcal{K}\left(\eta_{x}\right)-u u_{x} .
\end{aligned}
$$

Non-dimensionalizing (7) according to $\eta \rightarrow h_{0} \eta, u \rightarrow u \sqrt{g h_{0}}, x \rightarrow \alpha^{-1} h_{0} x, t \rightarrow$ $t \sqrt{h_{0} / g}$, and $c \rightarrow c \sqrt{g h_{0}}$ yields the following system:

$$
\begin{aligned}
& \alpha^{-1} \eta_{t}=c \eta_{x}-u_{x}-(\eta u)_{x} \\
& \alpha^{-1} u_{t}=c u_{x}-\mathcal{K}_{\alpha}\left(\eta_{x}\right)-u u_{x} .
\end{aligned}
$$

The parameter $\alpha$ is chosen to map $2 \pi / \kappa$-periodic solutions of (7) to $2 \pi$-periodic solutions of (8). Consequently, $\alpha=\kappa h_{0}>0$ and

$$
\widehat{\mathcal{K}_{\alpha}[f]}(n)=\frac{\tanh (\alpha n)}{\alpha n} \widehat{f}(n), \quad n \in \mathbb{Z},
$$

or, alternatively,

$$
\mathcal{K}_{\alpha}[f]=\left(\frac{\tanh (\alpha D)}{\alpha D}\right) f
$$

for $f \in \mathrm{L}_{\text {per }}^{1}(-\pi, \pi)$ with the Fourier transform (3) redefined over $(-\pi, \pi)$.

Stokes wave solutions of (8) are independent of time. Equating time derivatives in (8) to zero and integrating in $x$, we find

$$
\begin{aligned}
& c \eta=u+\eta u+\mathcal{I}_{1} \\
& c u=\mathcal{K}_{\alpha}(\eta)+\frac{1}{2} u^{2}+\mathcal{I}_{2},
\end{aligned}
$$

where $\mathcal{I}_{j}$ are integration constants. For each $\alpha>0$, there exists a three-parameter family of infinitely differentiable, even, small-amplitude, $2 \pi$-periodic solutions of (11), provided that $\mathcal{I}_{j}$ are sufficiently small [4]. We call these solutions the HPT-BW Stokes waves, denoted $\left(\eta_{S}\left(x ; \varepsilon, \mathcal{I}_{j}\right), u_{S}\left(x ; \varepsilon, \mathcal{I}_{j}\right)\right)^{T}$, where $\varepsilon$ is a small-amplitude parameter defined implicitly in terms of the first Fourier mode of $\eta_{S}\left(x ; \varepsilon, \mathcal{I}_{j}\right)$ :

$$
\varepsilon:=2 \widehat{\eta_{S}}(1)=\frac{1}{\pi} \int_{-\pi}^{\pi} \eta_{S}\left(x ; \varepsilon, \mathcal{I}_{j}\right) e^{-i x} d x .
$$

Remark 1. Redefining $c \rightarrow c-\mathcal{I}_{1}$ and $u \rightarrow u-\mathcal{I}_{1}$ in (11) implies $\mathcal{I}_{1}=0$ without loss of generality. If we also equate $\mathcal{I}_{2}=0$, our Stokes waves reduce to a one-parameter family of solutions to (11) such that (12) ensures that $\eta_{S}(x ; \varepsilon) \sim \varepsilon \cos (x)$ as $\varepsilon \rightarrow 0$. We restrict to this case for simplicitly, but the methodology in Sections 4 and 5 are unchanged if $\mathcal{I}_{2} \neq 0$. For series representations of Stokes waves that include $\mathcal{I}_{j}$, see [4].

The Stokes waves and their velocity may be expanded as power series in $\varepsilon$ :

$$
\begin{aligned}
& \eta_{S}=\eta_{S}(x ; \varepsilon)=\varepsilon \cos (x)+\sum_{j=2}^{\infty} \eta_{j}(x) \varepsilon^{j}, \\
& u_{S}=u_{S}(x ; \varepsilon)=c_{0} \varepsilon \cos (x)+\sum_{j=2}^{\infty} u_{j}(x) \varepsilon^{j}, \\
& c=c(\varepsilon)=c_{0}+\sum_{j=1}^{\infty} c_{2 j} \varepsilon^{2 j}, \quad c_{0}^{2}=\frac{\tanh (\alpha)}{\alpha},
\end{aligned}
$$


where $\eta_{j}(x)$ and $u_{j}(x)$ are analytic, even, and $2 \pi$-periodic for each $j$. Substituting these expansions into (11) (with $\mathcal{I}_{j}=0$ ) and following a Poincaré-Lindstedt perturbation method [2], one determines $\eta_{j}(x), u_{j}(x)$, and $c_{2 j}$ order by order. In Appendix A, we report expansions of $\eta_{S}, u_{S}$, and $c$ up to the fourth order in $\varepsilon$; this is sufficient for our asymptotic calculations of high-frequency isolas discussed in Sections 4 and 5.

\section{The Stability Spectrum of Stokes Waves}

We consider perturbations to $\left(\eta_{S}, u_{S}\right)^{T}$ of the form

$$
\left(\begin{array}{l}
\eta(x, t ; \varepsilon, \rho) \\
u(x, t ; \varepsilon, \rho)
\end{array}\right)=\left(\begin{array}{l}
\eta_{S}(x ; \varepsilon) \\
u_{S}(x ; \varepsilon)
\end{array}\right)+\rho\left(\begin{array}{l}
H(x, t ; \varepsilon) \\
U(x, t ; \varepsilon)
\end{array}\right)+\mathcal{O}\left(\rho^{2}\right)
$$

where $0<|\rho| \ll 1$ is a parameter independent of $\varepsilon$ and $H$, and $U$ are sufficiently smooth, bounded functions of $x$ on $\mathbb{R}$ for all $t \geq 0$. When (14) is substituted into (8), terms of $\mathcal{O}\left(\rho^{0}\right)$ cancel by (11) (with $\mathcal{I}_{j}=0$ ). Equating terms of $\mathcal{O}(\rho)$, the perturbation $(H, U)^{T}$ solves the linear system

$$
\frac{\partial}{\partial t}\left(\begin{array}{l}
H \\
U
\end{array}\right)=\alpha\left(\begin{array}{cc}
-u_{S}^{\prime}+\left(c-u_{S}\right) \partial_{x} & -\eta_{S}^{\prime}-\left(1+\eta_{S}\right) \partial_{x} \\
-\frac{i \tanh (\alpha D)}{\alpha} & -u_{S}^{\prime}+\left(c-u_{S}\right) \partial_{x}
\end{array}\right)\left(\begin{array}{l}
H \\
U
\end{array}\right)
$$

where primes denote differentiation with respect to $x$. Formally separating variables,

$$
\left(\begin{array}{l}
H(x, t ; \varepsilon) \\
U(x, t ; \varepsilon)
\end{array}\right)=e^{\lambda t}\left(\begin{array}{l}
\mathcal{H}(x, t ; \varepsilon) \\
\mathcal{U}(x, t ; \varepsilon)
\end{array}\right)
$$

where $(\mathcal{H}, \mathcal{U})^{T}$ solves the spectral problem

$$
\lambda\left(\begin{array}{l}
\mathcal{H} \\
\mathcal{U}
\end{array}\right)=\alpha\left(\begin{array}{cc}
-u_{S}^{\prime}+\left(c-u_{S}\right) \partial_{x} & -\eta_{S}^{\prime}-\left(1+\eta_{S}\right) \partial_{x} \\
-\frac{i \tanh (\alpha D)}{\alpha} & -u_{S}^{\prime}+\left(c-u_{S}\right) \partial_{x}
\end{array}\right)\left(\begin{array}{l}
\mathcal{H} \\
\mathcal{U}
\end{array}\right)
$$

Since the entries of the matrix operator above are $2 \pi$-periodic, one can use Floquet theory to solve $(17)$ for $(\mathcal{H}, \mathcal{U})^{T}$. (Strictly speaking, Floquet theory applies only to linear, local operators. Work by [23] extends this theory to nonlocal operators.) These solutions take the form

$$
\left(\begin{array}{l}
\mathcal{H}(x ; \varepsilon) \\
\mathcal{U}(x ; \varepsilon)
\end{array}\right)=e^{i \mu x}\left(\begin{array}{l}
\mathfrak{h}(x ; \varepsilon) \\
\mathfrak{u}(x ; \varepsilon)
\end{array}\right)
$$

where $\mu \in[-1 / 2,1 / 2]$ is called the Floquet exponent and $\mathfrak{h}, \mathfrak{u} \in \mathrm{H}_{\text {per }}^{1}(-\pi, \pi)$. Substituting (18) into (17) results in a spectral problem for $\mathbf{w}=(\mathfrak{h}, \mathfrak{u})^{\mathbf{T}}$ :

$$
\lambda_{\varepsilon, \mu} \mathbf{w}=\mathcal{L}_{\varepsilon, \mu} \mathbf{w},
$$

with

$$
\mathcal{L}_{\varepsilon, \mu}=\alpha\left(\begin{array}{cc}
-u_{S}^{\prime}+\left(c-u_{S}\right)\left(i \mu+\partial_{x}\right) & -\eta_{S}^{\prime}-\left(1+\eta_{S}\right)\left(i \mu+\partial_{x}\right) \\
-\frac{i \tanh (\alpha(\mu+D))}{\alpha} & -u_{S}^{\prime}+\left(c-u_{S}\right)\left(i \mu+\partial_{x}\right)
\end{array}\right) .
$$

For sufficiently small $\varepsilon,(19)$ has a countable collection of eigenvalues $\lambda_{\varepsilon, \mu}$ for each Floquet exponent $\mu$ [14]. The union of these eigenvalues over $\mu \in[-1 / 2,1 / 2]$ recovers the purely continuous spectrum of (17) for fixed $\varepsilon$; this is the stability spectrum of HPTBW Stokes waves. We use the Floquet-Fourier-Hill method [12] to compute the stability spectrum numerically.

If there exists a $\mu$ such that there is a $\lambda_{\varepsilon, \mu}$ with $\operatorname{Re}\left(\lambda_{\varepsilon, \mu}\right)>0$, then there exists a perturbation (16) that grows exponentially in time, and Stokes waves of amplitude $\varepsilon$ are spectrally unstable. If no such $\mu$ is found, then the Stokes waves are spectrally stable. 
Because of the quadrafold symmetry mentioned in the introduction, Stokes waves are spectrally stable if and only if their stability spectrum is a subset of the imaginary axis.

When $\varepsilon=0, \mathcal{L}_{0, \mu}$ has constant coefficients, and its spectral elements are given exactly by

$$
\lambda_{0, \mu, n}^{(\sigma)}=-i \Omega_{\sigma}(n+\mu), \quad n \in \mathbb{Z}, \quad \sigma= \pm 1,
$$

where $\Omega_{\sigma}$ are the two branches of the linear dispersion relation of (8) with $c \rightarrow c_{0}\left(c_{0}\right.$ is given in (13)). Explicitly,

$$
\Omega_{\sigma}(k)=-\alpha c_{0} k+\sigma \omega_{\alpha}(k)
$$

where

$$
\omega_{\alpha}(k)=\operatorname{sgn}(k) \sqrt{\alpha k \tanh (\alpha k)} .
$$

As expected, $\lambda_{0, \mu, n}^{(\sigma)}$ is a countable collection of eigenvalues for each $\mu$, and the resulting stability spectrum has quadrafold symmetry. In addition, the stability spectrum coincides with the imaginary axis, implying that zero-amplitude Stokes waves are spectrally stable.

For some $\mu=\mu_{0}$, nonzero eigenvalues of $\mathcal{L}_{0, \mu_{0}}$ with double multiplicity may give rise to Hamiltonian-Hopf bifurcations and, thus, to high-frequency instabilities for $0<|\varepsilon| \ll 1$. These eigenvalues exist, provided there exists $\mu_{0}, m$, and $n$, such that

$$
\lambda_{0, \mu_{0}, n}^{\left(\sigma_{1}\right)}=\lambda_{0, \mu_{0}, m}^{\left(\sigma_{2}\right)} \neq 0
$$

We view (24) as a collision of two simple, nonzero eigenvalues. It can be shown that such a collision occurs only if $\sigma_{1} \neq \sigma_{2}[3,4,24]$. Theorem A1 in Appendix B shows that, for any $p \in \mathbb{Z} \backslash\{0, \pm 1\}$, there exist unique $\mu_{0}, m$, and $n$ that satisfy (24) with $m-n=p$. Thus, there are a countably infinite number of nonzero eigenvalue collisions in the zeroamplitude stability spectrum; each of which has potential to develop a high-frequency instability in the small-amplitude stability spectrum.

Remark 2. Using results in Appendix B, it can be shown that the Krein signatures [25] of the colliding eigenvalues have opposite signs. This is a second necessary criterion for the occurance of high-frequency instabilities [3,26].

Remark 3. The WWP shares the same collided eigenvalues with HPT-BW, since (22) is also the dispersion relation of the WWP.

\section{High-Frequency Instabilities: $p=2$}

We use perturbation methods to investigate the high-frequency instability that develops from the collision of $\lambda_{0, \mu_{0}, n}^{(1)}$ and $\lambda_{0, \mu_{0}, m^{\prime}}^{(-1)}$ where $\mu_{0} \in[-1 / 2,1 / 2]$ is the unique Floquet exponent for which (24) is satisfied and $m-n=2$. (Because the spectrum (22) has the symmetry $\bar{\lambda}_{0,-\mu_{0},-n}^{(\sigma)}=\lambda_{0, \mu_{0}, n^{\prime}}^{(\sigma)}$ where the overbar denotes complex conjugation, choosing $p=-2$ gives the isola conjugate to that for $p=2$. Thus, we may choose $p=2$ without loss of generality.) This instability corresponds to the high-frequency isola closest to the origin; see Theorem A1 in Appendix B. For sufficiently small $\varepsilon$, this is also the isola with largest real component. 


\subsection{The $\mathcal{O}\left(\varepsilon^{0}\right)$ Problem}

The $p=2$ isola develops from the spectral data

$$
\begin{aligned}
\lambda_{0} & =\lambda_{0, \mu_{0}, n}^{(1)}=-i \Omega_{1}\left(\mu_{0}+n\right)=-i \Omega_{-1}\left(\mu_{0}+m\right)=\lambda_{0, \mu_{0}, m}^{(-1)} \neq 0, \\
\mathbf{w}_{\mathbf{0}}(x) & =\left(\begin{array}{c}
\mathfrak{h}_{0}(x) \\
\mathfrak{u}_{0}(x)
\end{array}\right)=\gamma_{0}\left(\begin{array}{c}
1 \\
-\frac{\omega_{\alpha}\left(m+\mu_{0}\right)}{\alpha\left(m+\mu_{0}\right)}
\end{array}\right) e^{i m x}+\gamma_{1}\left(\begin{array}{c}
1 \\
\frac{\omega_{\alpha}\left(n+\mu_{0}\right)}{\alpha\left(n+\mu_{0}\right)}
\end{array}\right) e^{i n x},
\end{aligned}
$$

where $\gamma_{j}$ are arbitrary, nonzero constants. As $|\varepsilon|$ increases, we assume the spectral data vary analytically [24] with $\varepsilon$ :

$$
\begin{aligned}
\lambda & =\lambda_{0}+\varepsilon \lambda_{1}+\varepsilon^{2} \lambda_{2}+\mathcal{O}\left(\varepsilon^{3}\right), \\
\mathbf{w} & =\mathbf{w}_{0}+\varepsilon \mathbf{w}_{\mathbf{1}}+\varepsilon^{2} \mathbf{w}_{2}+\mathcal{O}\left(\varepsilon^{3}\right) \\
& =\left(\begin{array}{l}
\mathfrak{h}_{0} \\
\mathfrak{u}_{0}
\end{array}\right)+\varepsilon\left(\begin{array}{l}
\mathfrak{h}_{1} \\
\mathfrak{u}_{1}
\end{array}\right)+\varepsilon^{2}\left(\begin{array}{l}
\mathfrak{h}_{2} \\
\mathfrak{u}_{2}
\end{array}\right)+\mathcal{O}\left(\varepsilon^{3}\right),
\end{aligned}
$$

where we suppress functional dependencies for ease of notation. We normalize $\mathbf{w}$ so that

$$
\widehat{\mathfrak{h}}(n)=\frac{1}{2 \pi} \int_{-\pi}^{\pi} \mathfrak{h} e^{-i n x} d x=1,
$$

or, alternatively, so that

$$
\begin{aligned}
& \widehat{\mathfrak{h}_{0}}(n)=1, \\
& \widehat{\mathfrak{h}_{j}}(n)=0, \quad \forall j \in \mathbb{N} .
\end{aligned}
$$

This normalization ensures that $\mathfrak{h}_{0}$ fully resolves the $n$th Fourier mode of $\mathfrak{h}$, a convenient choice for the perturbation calculations that follow. With this normalization,

$$
\mathbf{w}_{\mathbf{0}}(x)=\left(\begin{array}{l}
\mathfrak{h}_{0}(x) \\
\mathfrak{u}_{0}(x)
\end{array}\right)=\gamma_{0}\left(\begin{array}{c}
1 \\
-\frac{\omega_{\alpha}\left(m+\mu_{0}\right)}{\alpha\left(m+\mu_{0}\right)}
\end{array}\right) e^{i m x}+\left(\begin{array}{c}
1 \\
\frac{\omega_{\alpha}\left(n+\mu_{0}\right)}{\alpha\left(n+\mu_{0}\right)}
\end{array}\right) e^{i n x} .
$$

The arbitrary constant $\gamma_{0}$ will be determined at higher order, leading to a unique expression for $\mathbf{w}_{\mathbf{0}}$.

Remark 4. The eigenvalue corrections $\lambda_{j}$ derived below are independent of the normalization chosen for $\mathbf{w}$.

If $\lambda_{0}$ is a semi-simple, isolated eigenvalue of $\mathcal{L}_{0, \mu_{0}}$, we may justify (26) using analytic perturbation theory [27], provided the Floquet exponent is fixed. For sufficiently small $\varepsilon$, this method of proof gives two spectral elements on the isola. Numerical and asymptotic calculations show that these spectral elements quickly leave the isola as a result of the Floquet parameterization's dependence on $\varepsilon$ (Figure 2). To account for this dependence, we formally assume that the Floquet exponent depends analytically on $\varepsilon$ as well:

$$
\mu=\mu_{0}+\varepsilon \mu_{1}+\varepsilon^{2} \mu_{2}+\mathcal{O}\left(\varepsilon^{3}\right) .
$$

Remark 5. In the calculations that follow, explicit expressions of select quantities are suppressed for ease of readability. The interested reader may consult the file in Data Availability Statement hptbw_isolap2.nb for these expressions. 


\subsection{The $\mathcal{O}(\varepsilon)$ Problem}

Substituting the expansions of the Stokes wave (13), spectral data (26), and Floquet exponent (30) into the spectral problem (19) and collecting terms of $\mathcal{O}(\varepsilon)$, we find

$$
\left(\mathcal{L}_{0, \mu_{0}}-\lambda_{0}\right) \mathbf{w}_{\mathbf{1}}=\lambda_{1} \mathbf{w}_{\mathbf{0}}-\mathcal{L}_{1} \mathbf{w}_{\mathbf{0}},
$$

with

$$
\mathcal{L}_{1}=\alpha\left(\begin{array}{cc}
-u_{1}^{\prime}+i c_{0} \mu_{1}-u_{1}\left(i \mu_{0}+\partial_{x}\right) & -\eta_{1}^{\prime}-i \mu_{1}-\eta_{1}\left(i \mu_{0}+\partial_{x}\right) \\
-i \mu_{1} \operatorname{sech}^{2}\left(\alpha\left(\mu_{0}+D\right)\right) & -u_{1}^{\prime}+i c_{0} \mu_{1}-u_{1}\left(i \mu_{0}+\partial_{x}\right)
\end{array}\right)
$$

The inhomogeneous terms on the RHS of (31) can be evaluated using expressions for $\eta_{1}, u_{1}$, and $\mathbf{w}_{0}$. Each of these quantities are finite linear combinations of $2 \pi$-periodic sinusoids. As a result, the inhomogeneous terms can be rewritten as a finite Fourier series, and (31) becomes

$$
\left(\mathcal{L}_{0, \mu_{0}}-\lambda_{0}\right) \mathbf{w}_{\mathbf{1}}=\sum_{j=n-1}^{m+1} \mathbf{T}_{\mathbf{1}, \mathrm{j}} e^{i j x}
$$

where $\mathbf{T}_{\mathbf{1}, \mathbf{j}}$ depend on $\mu_{0}, \alpha$, and $\gamma_{0}$; see the file in Data Availability Statement for details.

Remark 6. Since $m-n=2$, the index $j \in\{n-1, n, 1+n, m, m+1\}$. When evaluating the inhomogeneous terms, one finds vector multiples of $\exp (i(1+n) x)$ and $\exp (i(m-1) x)$. These vectors are combined to give $\mathbf{T}_{\mathbf{1 , 1 + n}}$.

For (33) to have a solution $\mathbf{w}_{\mathbf{1}}$, the inhomogeneous terms must be orthogonal (in the $\mathrm{L}_{\text {per }}^{2}(-\pi, \pi) \times \mathrm{L}_{\text {per }}^{2}(-\pi, \pi)$ sense) to the nullspace of the hermitian adjoint of $\mathcal{L}_{0, \mu_{0}}-\lambda_{0}$ by the Fredholm alternative. The hermitian adjoint of $\mathcal{L}_{0, \mu_{0}}-\lambda_{0}$ is

$$
\left(\mathcal{L}_{0, \mu_{0}}-\lambda_{0}\right)^{\dagger}=\left(\begin{array}{cc}
-\alpha c_{0}\left(i \mu_{0}+\partial_{x}\right)-\overline{\lambda_{0}} & i \tanh \left(\alpha\left(\mu_{0}+D\right)\right) \\
\alpha\left(i \mu_{0}+\partial_{x}\right) & -\alpha c_{0}\left(i \mu_{0}+\partial_{x}\right)-\overline{\lambda_{0}}
\end{array}\right),
$$

where overbars denote complex conjugation. Its nullspace is

$$
\operatorname{Null}\left[\left(\mathcal{L}_{0, \mu_{0}}-\lambda_{0}\right)^{\dagger}\right]=\operatorname{Span}\left[\left(\begin{array}{c}
1 \\
\frac{\alpha\left(\mu_{0}+n\right)}{\omega_{\alpha}\left(\mu_{0}+n\right)}
\end{array}\right) e^{i n x},\left(\begin{array}{c}
1 \\
-\frac{\alpha\left(\mu_{0}+m\right)}{\omega_{\alpha}\left(\mu_{0}+m\right)}
\end{array}\right) e^{i m x}\right] .
$$

Thus, according to the Fredholm alternative, there exists a solution $\mathbf{w}_{\mathbf{1}}$ to (33) if

$$
\left\langle\left(\begin{array}{c}
1 \\
\frac{\alpha\left(\mu_{0}+n\right)}{\omega_{\alpha}\left(\mu_{0}+n\right)}
\end{array}\right) e^{i n x}, \mathbf{T}_{\mathbf{1}, \mathbf{n}} e^{i n x}\right\rangle=0, \quad\left\langle\left(\begin{array}{c}
1 \\
-\frac{\alpha\left(\mu_{0}+m\right)}{\omega_{\alpha}\left(\mu_{0}+m\right)}
\end{array}\right) e^{i m x}, \mathbf{T}_{\mathbf{1}, \mathbf{m}} e^{i m x}\right\rangle=0,
$$

where $\langle\cdot, \cdot\rangle$ is the standard inner product on $\mathrm{L}_{\text {per }}^{2}(-\pi, \pi) \times \mathrm{L}_{\text {per }}^{2}(-\pi, \pi)$. Substituting expressions for $\mathbf{T}_{\mathbf{1 , n}}$ and $\mathbf{T}_{\mathbf{1}, \mathrm{m}}$ gives solvability conditions

$$
\begin{array}{r}
\lambda_{1}+i \mu_{1} c_{g_{1}}\left(\mu_{0}+n\right)=0 \\
\gamma_{0}\left(\lambda_{1}+i \mu_{1} c_{g_{-1}}\left(\mu_{0}+m\right)\right)=0,
\end{array}
$$

where $c_{g_{\sigma}}(k)=\Omega_{\sigma}^{\prime}(k)$ is the group velocity of $\Omega_{\sigma}$. Lemma A3 in Appendix B shows that $c_{g_{1}}\left(\mu_{0}+n\right) \neq c_{g_{-1}}\left(\mu_{0}+m\right)$. Since $\gamma_{0}$ is nonzero,

$$
\lambda_{1}=0=\mu_{1} .
$$

Consequently, $\mathbf{T}_{\mathbf{1}, \mathbf{n}}=\mathbf{0}=\mathbf{T}_{\mathbf{1}, \mathrm{m}}$, simplifying the inhomogeneous terms in (33). 
With the solvability conditions satisfied, we solve for the particular solution of $\mathbf{w}_{\mathbf{1}}$ in (33). Combining with the nullspace of $\mathcal{L}_{0, \mu_{0}}-\lambda_{0}$,

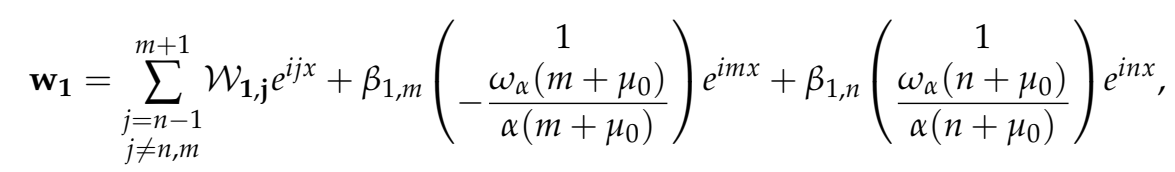

where $\beta_{1, j}$ are arbitrary constants and $\mathcal{W}_{\mathbf{1}, \mathrm{j}}$ are found in the file in Data Availability Statement. Enforcing the normalization condition (28), one finds $\beta_{1, n}=0$. For ease of notation, let $\beta_{1, m} \rightarrow \gamma_{1}$ so that

$$
\mathbf{w}_{\mathbf{1}}=\sum_{\substack{j=n-1 \\
j \neq n, m}}^{m+1} \mathcal{W}_{\mathbf{1}, \mathrm{j}} \mathrm{e}^{i j x}+\gamma_{1}\left(\begin{array}{c}
1 \\
-\frac{\omega_{\alpha}\left(m+\mu_{0}\right)}{\alpha\left(m+\mu_{0}\right)}
\end{array}\right) e^{i m x}
$$

\subsection{The $\mathcal{O}\left(\varepsilon^{2}\right)$ Problem}

Using (38), the spectral problem (19) at $\mathcal{O}\left(\varepsilon^{2}\right)$ is

$$
\left(\mathcal{L}_{0, \mu_{0}}-\lambda_{0}\right) \mathbf{w}_{\mathbf{2}}=\lambda_{2} \mathbf{w}_{\mathbf{0}}-\left.\mathcal{L}_{2}\right|_{\mu_{1}=0} \mathbf{w}_{\mathbf{0}}-\left.\mathcal{L}_{1}\right|_{\mu_{1}=0} \mathbf{w}_{\mathbf{1}},
$$

where $\left.\mathcal{L}_{1}\right|_{\mu_{1}=0}$ is the same as above, but evaluated at $\mu_{1}=0$, and

$$
\left.\mathcal{L}_{2}\right|_{\mu_{1}=0}=\alpha\left(\begin{array}{cc}
-u_{2}^{\prime}+\left(c_{2}-u_{2}\right)\left(i \mu_{0}+\partial_{x}\right)+i \mu_{2} c_{0} & -\eta_{2}^{\prime}-i \mu_{2}-\eta_{2}\left(i \mu_{0}+\partial_{x}\right) \\
-i \mu_{2} \operatorname{sech}^{2}\left(\alpha\left(\mu_{0}+D\right)\right) & -u_{2}^{\prime}+\left(c_{2}-u_{2}\right)\left(i \mu_{0}+\partial_{x}\right)+i \mu_{2} c_{0}
\end{array}\right) .
$$

One can evaluate the inhomogeneous terms of (42) using $\eta_{j}, u_{j}$, and $\mathbf{w}_{\mathbf{j}-\mathbf{1}}$ for $j \in\{1,2\}$. These inhomogeneous terms can be expressed as a finite Fourier series, giving

$$
\left(\mathcal{L}_{0, \mu_{0}}-\lambda_{0}\right) \mathbf{w}_{2}=\sum_{\substack{j=n-2 \\ j \neq n-1}}^{m+2} \mathbf{T}_{2, j} e^{i j x}
$$

It can be shown that $\mathbf{T}_{\mathbf{2 , n}-\mathbf{1}}=\mathbf{0}$.

Proceeding similarly to the previous order, solvability conditions for (43) are

$$
\begin{aligned}
2\left(\lambda_{2}+i \mathcal{C}_{1, n}\right)+i \gamma_{0} \mathcal{S}_{2, n} & =0, \\
2 \gamma_{0}\left(\lambda_{2}+i \mathcal{C}_{-1, m}\right)+i \mathcal{S}_{2, m} & =0,
\end{aligned}
$$

where

$$
\begin{aligned}
\mathcal{C}_{1, n} & =\mu_{2} c_{g_{1}}\left(\mu_{0}+n\right)-\mathcal{P}_{2, n}, \\
\mathcal{C}_{-1, m} & =\mu_{2} c_{g_{-1}}\left(\mu_{0}+m\right)-\mathcal{P}_{2, m} .
\end{aligned}
$$

Expressions for $\mathcal{S}_{2, j}$ and $\mathcal{P}_{2, j}$ have no dependence on $\gamma_{0}, \gamma_{1}, \mu_{2}$, or $\lambda_{2}$; see the file in Data Availability Statement for details.

Conditions (44a) and (44b) form a nonlinear system for $\gamma_{0}$ and $\lambda_{2}$. Solving for $\lambda_{2}$ yields

$$
\lambda_{2}=-i\left(\frac{\mathcal{C}_{-1, m}+\mathcal{C}_{1, n}}{2}\right) \pm \sqrt{-\left(\frac{\mathcal{C}_{-1, m}-\mathcal{C}_{1, n}}{2}\right)^{2}+\frac{\mathcal{S}_{2}^{2}}{4 \omega_{\alpha}\left(\mu_{0}+m\right) \omega_{\alpha}\left(\mu_{0}+n\right)}}
$$

where

$$
\mathcal{S}_{2, n} \mathcal{S}_{2, m}=-\frac{\mathcal{S}_{2}^{2}}{\omega_{\alpha}\left(\mu_{0}+m\right) \omega_{\alpha}\left(\mu_{0}+n\right)}
$$


A corollary of Lemma A3 in Appendix B shows that $\omega_{\alpha}\left(\mu_{0}+m\right) \omega_{\alpha}\left(\mu_{0}+n\right)$ is positive. (This corollary is equivalent to satisfying the Krein signature condition mentioned in Section 3) Provided $\mathcal{S}_{2} \neq 0$ and $c_{g_{-1}}\left(\mu_{0}+m\right) \neq c_{g_{1}}\left(\mu_{0}+n\right), \lambda_{2}$ has nonzero real part for $\mu_{2} \in\left(M_{2,-}, M_{2,+}\right)$, where

$$
M_{2, \pm}=\mu_{2, *} \pm \frac{\left|\mathcal{S}_{2}\right|}{\left|c_{g_{-1}}\left(\mu_{0}+m\right)-c_{g_{1}}\left(\mu_{0}+n\right)\right| \sqrt{\omega_{\alpha}\left(\mu_{0}+m\right) \omega_{\alpha}\left(\mu_{0}+n\right)}},
$$

and

$$
\mu_{2, *}=\frac{\mathcal{P}_{2, m}-\mathcal{P}_{2, n}}{c_{g_{-1}}\left(\mu_{0}+m\right)-c_{g_{1}}\left(\mu_{0}+n\right)} .
$$

That $c_{g_{-1}}\left(\mu_{0}+m\right) \neq c_{g_{1}}\left(\mu_{0}+n\right)$ follows from Lemma A2 in Appendix B. A plot of $\mathcal{S}_{2}$ as a function of $\alpha$ suggests that $\mathcal{S}_{2}>0$ for all values of $\alpha>0$ (Figure 3). We conjecture that HPT-BW Stokes waves of any wavenumer experience a $p=2$ highfrequency instability at $\mathcal{O}\left(\varepsilon^{2}\right)$.

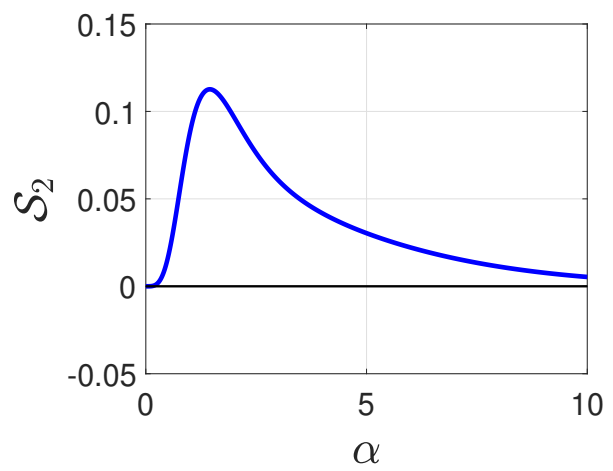

Figure 3. A plot of $\mathcal{S}_{2}$ vs. $\alpha$. No roots of $\mathcal{S}_{2}$ are found for $\alpha>0$. It is likely that the Hur-Pandey-TaoBoussinesq-Whitham (HPT-BW) Stokes waves of all wavenumbers experience a $p=2$ instability.

For $\mu_{2} \in\left(M_{2,-}, M_{2,+}\right)$, a quick calculation shows that (46) parameterizes an ellipse asymptotic to the numerically observed $p=2$ high-frequency isola (Figure 4 ). The ellipse has semi-major and -minor axes that scale like $\varepsilon^{2}$, and the center of the ellipse drifts along the imaginary axis from $\lambda_{0}$ like $\varepsilon^{2}$.

The midpoint of $\left(M_{2,-}, M_{2,+}\right)$ maximizes the real part of $\lambda_{2}$. Thus, the most unstable spectral element of the isola has Floquet exponent

$$
\mu_{*}=\mu_{0}+\mu_{2, *} \varepsilon^{2}+\mathcal{O}\left(\varepsilon^{3}\right),
$$

and its real and imaginary components are

$$
\begin{aligned}
& \lambda_{r, *}=\left(\frac{\left|\mathcal{S}_{2}\right|}{2 \sqrt{\omega_{\alpha}\left(\mu_{0}+m\right) \omega_{\alpha}\left(\mu_{0}+n\right)}}\right) \varepsilon^{2}+\mathcal{O}\left(\varepsilon^{3}\right), \\
& \lambda_{i, *}=-\Omega_{1}\left(\mu_{0}+n\right)-\mathcal{C}_{1, n} \varepsilon^{2}+\mathcal{O}\left(\varepsilon^{3}\right),
\end{aligned}
$$

respectively. These expansions agree well with the FFH results (Figure 5). 

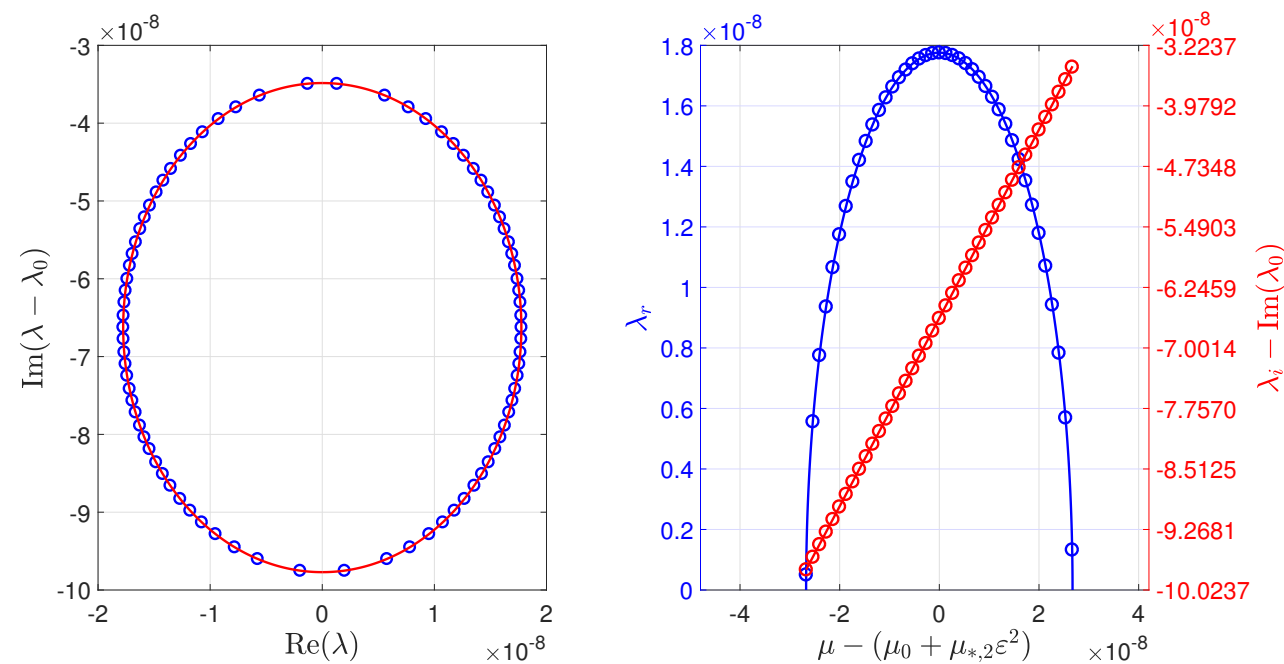

Figure 4. (Left) The $p=2$ isola with $\alpha=1$ and $\varepsilon=5 \times 10^{-4}$. The zeroth-order imaginary correction is removed for better visibility. The solid red curve is the ellipse obtained by our perturbation calculations. Blue circles are a subset of spectral elements from the numerically computed isola using loquet-Fourier-Hill (FFH). (Right) The Floquet parameterization of the real (blue) and imaginary (red) components of the isola. Both the zeroth- and second-order corrections of the Floquet exponent and the zeroth-order correction of the imaginary component are removed for better visibility. Solid curves illustrate perturbation results. Circles indicate FFH results.
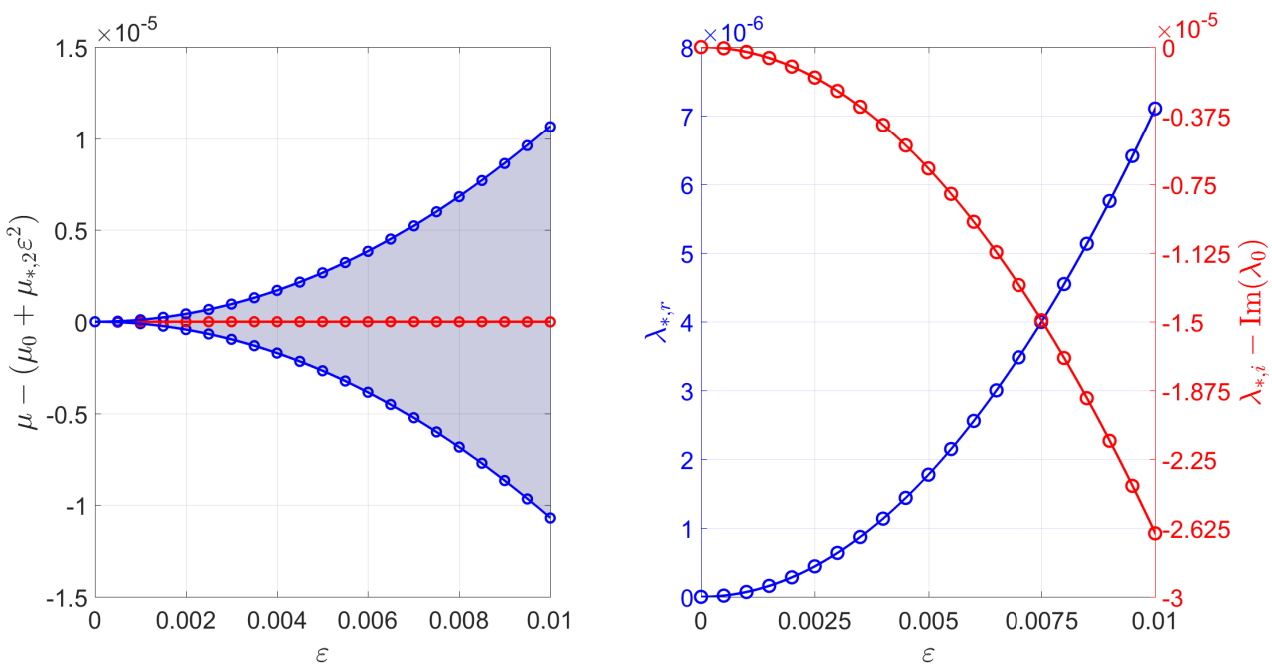

Figure 5. (Left) The interval of Floquet exponents that parameterize the $p=2$ isola as a function of $\varepsilon$ for $\alpha=1$. The zeroth- and second-order Floquet corrections are removed for better visibility. Up to recentering the Floquet axis and accounting for a wider range of $\varepsilon$, this plot is identical to the right panel of Figure 2. Solid blue curves indicate the boundaries of this interval according to our perturbation calculations. Blue circles indicate the boundaries computed numerically by FFH. The solid red curve gives the Floquet exponent corresponding to the most unstable spectral element of the isola according to our perturbation calculations. Red circles indicate the same but computed numerically using FFH. (Right) The real (blue) and imaginary (red) components of the most unstable spectral element of the isola as a function of $\varepsilon$. The zeroth-order imaginary correction is removed for better visibility. Solid curves illustrate perturbation calculations. Circles illustrate FFH results. 


\section{High-Frequency Instabilities: $p=3$}

According to Theorem A1 in Appendix B, the $p=3$ high-frequency instability is the second-closest to the origin. This instability arises at $\mathcal{O}\left(\varepsilon^{3}\right)$. Let $\mu_{0}$ correspond to the unique Floquet exponent in $[-1 / 2,1 / 2]$ that satisfies the collision condition (24) with $m-n=3$. Then, the spectral data (25) give rise to the $p=3$ high-frequency instability. We assume these data and the Floquet exponent vary analytically with $\varepsilon$. For uniqueness, we normalize the eigenfunction $\mathbf{w}$ according to (28) so that $\mathbf{w}_{\mathbf{0}}$ is given by (29).

The calculations proceed similarly as in the $p=2$ case, with two major exceptions. (i) At $\mathcal{O}\left(\varepsilon^{2}\right)$, the solvability conditions are linear in $\lambda_{2}$ and $\mu_{2}$ and independent of $\gamma_{0}$, leading only to a purely imaginary correction to the isola at this order. (ii) At $\mathcal{O}\left(\varepsilon^{3}\right)$, the solvability conditions depend nonlinearly on $\lambda_{3}$ and $\gamma_{0}$, but also include a term proportional to $\gamma_{1}$. One can show that this term vanishes due to the solvability conditions at $\mathcal{O}\left(\varepsilon^{2}\right)$. For more details, see Appendix C.

Remark 7. In Appendix $C$ and what follows, explicit expressions of select quantities are suppressed for ease of readability. Some quantities borrow notation from the $p=2$ case (but evaluate differently). The interested reader may consult the file in Data Availability Statement hptbw_isolap3.nb for these expressions.

Solving for $\lambda_{3}$ in the solvability conditions at $\mathcal{O}\left(\varepsilon^{3}\right)$, one finds

$$
\begin{aligned}
\lambda_{3}= & -i \mu_{3}\left(\frac{c_{g_{-1}}\left(\mu_{0}+m\right)+c_{g_{1}}\left(\mu_{0}+n\right)}{2}\right) \\
& \pm \sqrt{-\mu_{3}^{2}\left(\frac{c_{g_{-1}}\left(\mu_{0}+m\right)-c_{g_{1}}\left(\mu_{0}+n\right)}{2}\right)^{2}+\frac{\mathcal{S}_{3}^{2}}{4 \omega_{\alpha}\left(\mu_{0}+m\right) \omega_{\alpha}\left(\mu_{0}+n\right)}},
\end{aligned}
$$

where $\mathcal{S}_{3}$ is given in the file in Data Availability Statement. As in the $p=2$ case, $\omega_{\alpha}\left(\mu_{0}+\right.$ $m) \omega_{\alpha}\left(\mu_{0}+n\right)>0$ and $c_{g_{-1}}\left(\mu_{0}+m\right) \neq c_{g_{1}}\left(\mu_{0}+n\right)$. Provided $\mathcal{S}_{3} \neq 0, \lambda_{3}$ has nonzero real part if $\mu_{3} \in\left(-M_{3}, M_{3}\right)$, where

$$
M_{3}=\frac{\left|\mathcal{S}_{3}\right|}{\left|c_{g_{-1}}\left(\mu_{0}+m\right)-c_{g_{1}}\left(\mu_{0}+n\right)\right| \sqrt{\omega_{\alpha}\left(\mu_{0}+m\right) \omega_{\alpha}\left(\mu_{0}+n\right)}} .
$$

A plot of $\mathcal{S}_{3}$ vs. $\alpha$ reveals that $\mathcal{S}_{3}=0$ only at $\alpha=1.1862 \ldots$ (Figure 6). For this aspect ratio, the $p=3$ instability does not occur at $\mathcal{O}\left(\varepsilon^{3}\right)$. In fact, Figure 6 shows that, if $\alpha$ approaches $1.1862 \ldots$. for fixed $\varepsilon$, the numerically computed $p=3$ isola shrinks to a point on the imaginary axis. We conjecture that HPT-BW Stokes waves with $\alpha=1.1862 \ldots$ do not have a $p=3$ instability, even beyond $\mathcal{O}\left(\varepsilon^{3}\right)$. In the next subsection, we find that $\lambda_{4}$ is purely imaginary, so Stokes waves with aspect ratio $\alpha=1.1862 \ldots$ do not exhibit $p=3$ instabilities to $\mathcal{O}\left(\varepsilon^{4}\right)$.

Assuming $\alpha \neq 1.1862 \ldots, \mu_{3} \in\left(-M_{3}, M_{3}\right)$ parameterizes an ellipse asymptotic to the $p=3$ high-frequeny isola; see Figure 7. The ellipse has semi-major and -minor axes that scale like $\varepsilon^{3}$. The center of this ellipse drifts along the imaginary axis like $\varepsilon^{2}$ due to the purely imaginary correction found at $\mathcal{O}\left(\varepsilon^{2}\right)$. 

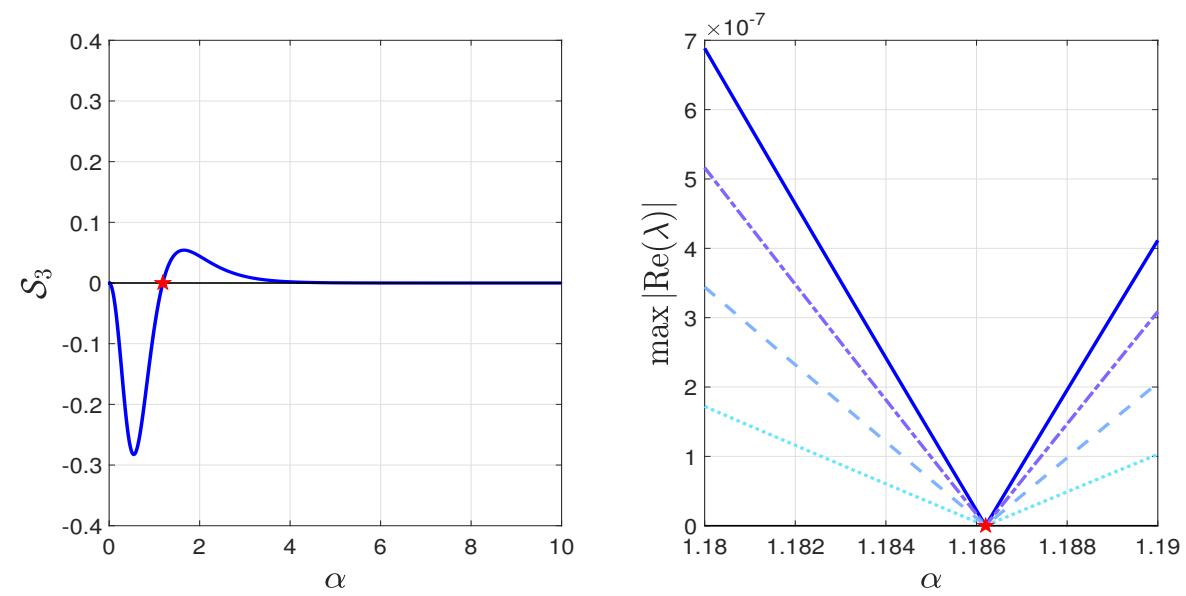

Figure 6. (Left) A plot of $\mathcal{S}_{3}$ vs. $\alpha$. The quantity $\mathcal{S}_{3}$ has a root $\alpha=1.1862 \ldots$ (red star), implying that HPBT-BW Stokes waves of this aspect ratio do not have a $p=3$ instability at $\mathcal{O}\left(\varepsilon^{3}\right)$. (Right) A plot of the maximum real component of the numerical $p=3$ isola (computed by FFH) as a function of $\alpha$ for $\varepsilon=10^{-3}$ (solid blue), $\varepsilon=7.5 \times 10^{-4}$ (dot-dashed purple), $\varepsilon=5 \times 10^{-4}$ (dashed light blue), and $\varepsilon=2.5 \times 10^{-4}$ (dotted cyan). The $p=3$ isola vanishes when $\alpha=1.1862 \ldots$ (red star).
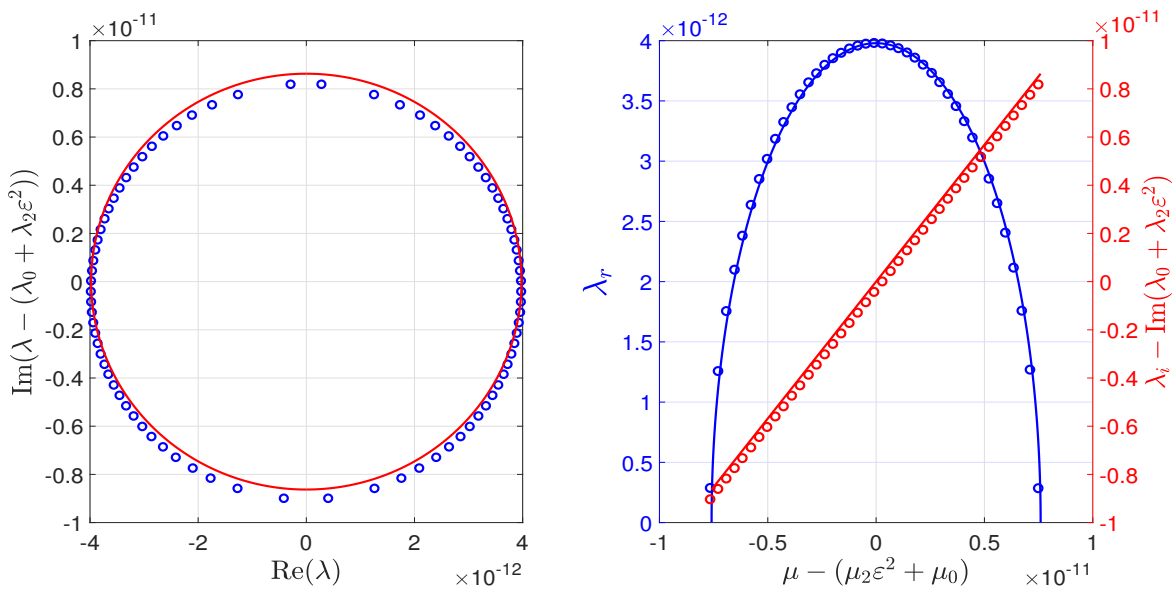

Figure 7. (Left) The $p=3$ isola with $\alpha=1$ and $\varepsilon=5 \times 10^{-4}$. The zeroth- and second-order imaginary corrections are removed for better visibility. The solid red curve is the ellipse obtained by our perturbation calculations. The blue circles are a subset of spectral elements from the numerically computed isola using FFH. (Right) The Floquet parameterization of the real (blue) and imaginary (red) components of the isola. Both the zeroth- and second-order corrections of the imaginary component and Floquet exponent are removed for better visibility. Solid curves illustrate perturbation results. Circles indicate FFH results.

The interval of Floquet exponents that parameterizes the $p=3$ isola is

$$
\mu \in\left(\mu_{0}+\mu_{2} \varepsilon^{2}-M_{3} \varepsilon^{3}, \mu_{0}+\mu_{2} \varepsilon^{2}+M_{3} \varepsilon^{3}\right)+\mathcal{O}\left(\varepsilon^{4}\right),
$$

where $\mu_{2}$ is given by (A23b). The width of this interval is an order of magnitude smaller than that of the $p=2$ isola. Consequently, the $p=3$ isola is more challenging to find numerically than the $p=2$ isola, at least for methods similar to FFH (Table 1 ).

For $\alpha=1$ and $|\varepsilon|<5 \times 10^{-4}$, (54) provides an excellent approximation to the numerically computed interval of Floquet exponents (Figure 8). Fourth-order corrections are necessary to improve agreement between (54) and numerical computations for larger $\varepsilon$, as is done below. 
Table 1. Intervals of Floquet exponents that parameterize the $p=2$ and $p=3$ high-frequency isolas with $\varepsilon=10^{-3}$ and $\alpha=1 / 2,1$, and 2 . The first digit for which the boundary values disagree is underlined and colored red. If a uniform mesh of Floquet exponents in $[-1 / 2,1 / 2]$ is used for numerical methods like $\mathrm{FFH}$, the spacing of the mesh must be finer than $\varepsilon^{2}$ to capture the $p=2$ instability and $\varepsilon^{3}$ to capture the $p=3$ instability. The intervals vary with $\alpha$ as well, making it difficult to adapt and refine a uniform mesh to find high-frequency isolas.

\begin{tabular}{cc}
\hline & $p=2$ \\
\hline$\alpha=\frac{1}{2}$ & $(-0.106478 \underline{8} 13547533,-0.106478 \underline{6} 33575956)$ \\
$\alpha=1$ & $(-0.26090 \underline{9} 131823605,-0.26090 \underline{8} 917941151)$ \\
$\alpha=2$ & $(-0.330352 \underline{196060556,-0.3303522275321770)}$ \\
\hline$\alpha=\frac{1}{2}$ & $p=3$ \\
$\alpha=1$ & $(-0.375448877 \underline{0} 009085,-0.37544887 \underline{5} 412116)$ \\
$\alpha=2$ & $(0.257196721 \underline{100572}, 0.257196721 \underline{3} 43587)$ \\
\end{tabular}

Choosing $\mu_{3}=0$ maximizes the real part of $\lambda_{3}$. Thus, the most unstable spectral element of the $p=3$ isola has Floquet exponent

$$
\mu_{*}=\mu_{0}+\mu_{2} \varepsilon^{2}+\mathcal{O}\left(\varepsilon^{4}\right),
$$

where $\mu_{2}$ is as in (A23b), and its real and imaginary components are

$$
\begin{aligned}
& \lambda_{r, *}=\left(\frac{\left|\mathcal{S}_{3}\right|}{2 \sqrt{\omega_{\alpha}\left(\mu_{0}+m\right) \omega_{\alpha}\left(\mu_{0}+n\right)}}\right) \varepsilon^{3}+\mathcal{O}\left(\varepsilon^{4}\right), \\
& \lambda_{i, *}=-\Omega_{1}\left(\mu_{0}+n\right)-\left(\frac{\mathcal{P}_{2, m} c_{g_{1}}\left(\mu_{0}+n\right)-\mathcal{P}_{2, n} c_{g_{-1}}\left(\mu_{0}+m\right)}{c_{g_{-1}}\left(\mu_{0}+m\right)-c_{g_{1}}\left(\mu_{0}+n\right)}\right) \varepsilon^{2}+\mathcal{O}\left(\varepsilon^{4}\right),
\end{aligned}
$$

respectively. Expressions for $\mathcal{P}_{2, n}$ and $\mathcal{P}_{2, m}$ are found in the file in Data Availability Statement. The expansion for $\lambda_{r, *}$ is in excellent agreement with numerical results using the FFH method (Figure 8). As with (54), corrections to $\mu_{*}$ and $\lambda_{i, *}$ at $\mathcal{O}\left(\varepsilon^{4}\right)$ improve the agreement between numerical and asymptotic results for these quantities.

Before proceeding to $\mathcal{O}\left(\varepsilon^{4}\right)$, we solve the $\mathcal{O}\left(\varepsilon^{3}\right)$ problem (Equation (A27) in Appendix C), subject to the solvability and normalization conditions. We find

$$
\mathbf{w}_{\mathbf{3}}=\sum_{\substack{j=n-3 \\
j \neq n-2}}^{m+3} \mathcal{W}_{3, j} \mathrm{e}^{i j x}+\gamma_{3}\left(\begin{array}{c}
1 \\
-\frac{\omega_{\alpha}\left(m+\mu_{0}\right)}{\alpha\left(m+\mu_{0}\right)} e^{i m x}
\end{array}\right),
$$

where $\gamma_{3}$ is arbitrary, $\mathcal{W}_{3, \mathbf{n}-2}=\mathbf{0}$, and the remaining $\mathcal{W}_{3, \mathbf{j}}$ are in the file in Data Availability Statement.

\section{The $\mathcal{O}\left(\varepsilon^{4}\right)$ Problem}

In this section, we derive corrections to the $p=3$ isola beyond its leading-order behavior. As will be seen, a new condition must be introduced to close the solvability conditions at this order. This extends the perturbation method introduced in [22]. We anticipate that our extension generalizes for corrections beyond the leading-order behavior of any isola. 

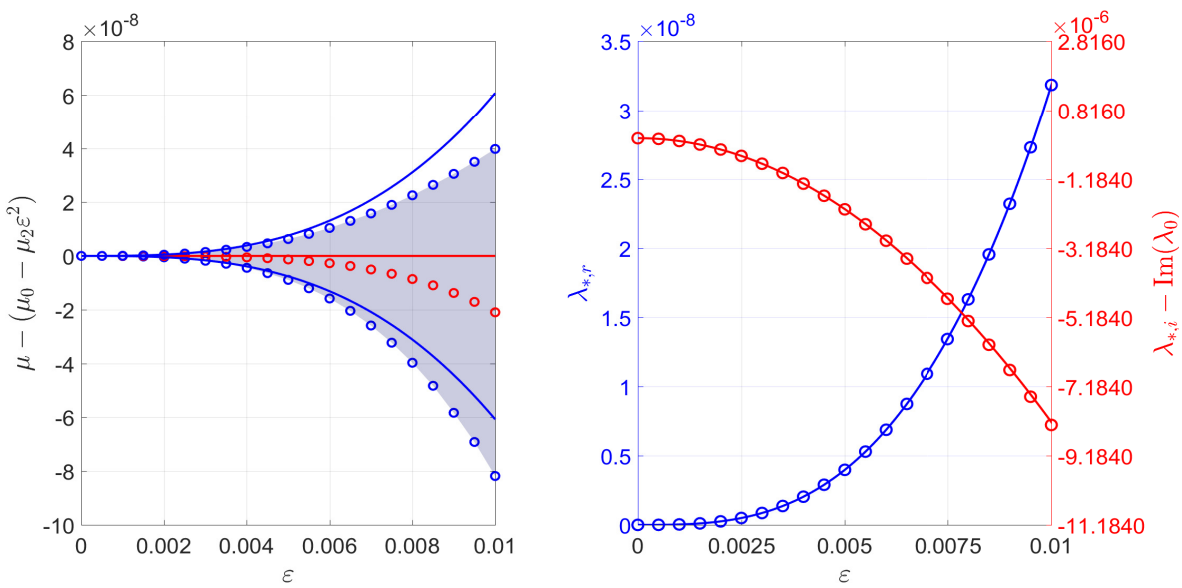

Figure 8. (Left) The interval of Floquet exponents that parameterize the $p=3$ isola as a function of $\varepsilon$ for $\alpha=1$. The zeroth- and second-order Floquet corrections are removed for better visibility. Solid blue curves indicate the boundaries of this interval according to our perturbation calculations. Blue circles indicate the boundaries computed numerically by FFH. The solid red curve gives the Floquet exponent corresponding to the most unstable spectral element of the isola according to our perturbation calculations. Red circles indicate the same but computed numerically using FFH. (Right) The real (blue) and imaginary (red) components of the most unstable spectral element of the isola as a function of $\varepsilon$. The zeroth- and second-order corrections of both the imaginary component and Floquet exponent are removed for better visibility. Solid curves illustrate perturbation calculations. Circles illustrate FFH results.

$\operatorname{At} \mathcal{O}\left(\varepsilon^{4}\right)$

$$
\left(\mathcal{L}_{0, \mu_{0}}-\lambda_{0}\right) \mathbf{w}_{\mathbf{4}}=\sum_{j=2}^{4} \lambda_{j} \mathbf{w}_{\mathbf{3}-\mathbf{j}}-\left.\sum_{j=1}^{4} \mathcal{L}_{j}\right|_{\mu_{1}=0} \mathbf{w}_{\mathbf{3}-\mathbf{j}}
$$

where $\left.\mathcal{L}_{j}\right|_{\mu_{1}=0}$ are as before and

$$
\left.\mathcal{L}_{4}\right|_{\mu_{1}=0}=\alpha\left(\begin{array}{cc}
\mathcal{L}_{4}^{(1,1)} & \mathcal{L}_{4}^{(1,2)} \\
\mathcal{L}_{4}^{(2,1)} & \mathcal{L}_{4}^{(1,1)}
\end{array}\right)
$$

with

$$
\begin{aligned}
& \mathcal{L}_{4}^{(1,1)}=i c_{0} \mu_{4}-i \mu_{3} u_{1}+i \mu_{2}\left(c_{2}-u_{2}\right)+\left(c_{4}-u_{4}\right)\left(i \mu_{0}+\partial_{x}\right)-u_{4}^{\prime} \\
& \mathcal{L}_{4}^{(1,2)}=-i \mu_{4}-i \mu_{3} \eta_{1}-i \mu_{2} \eta_{2}-\eta_{4}\left(i \mu_{0}+\partial_{x}\right)-\eta_{4^{\prime}}^{\prime} \\
& \mathcal{L}_{4}^{(2,1)}=-i \mu_{4} \operatorname{sech}^{2}\left(\alpha\left(\mu_{0}+D\right)\right)+i \alpha \mu_{2}^{2} \operatorname{sech}\left(\alpha\left(\mu_{0}+D\right)\right) \tanh \left(\alpha\left(\mu_{0}+D\right)\right) .
\end{aligned}
$$

Substituting $\eta_{j}, u_{j}$, and $\mathbf{w}_{\mathbf{j}-\mathbf{1}}$ for $j \in\{1,2,3\}$ into (58), we find

$$
\left(\mathcal{L}_{0, \mu_{0}}-\lambda_{0}\right) \mathbf{w}_{\mathbf{4}}=\sum_{\substack{j=n-4 \\ j \neq n-3}}^{m+4} \mathbf{T}_{\mathbf{4}, \mathbf{j}} e^{i j x}
$$

where $\mathbf{T}_{4, \mathbf{n}-3}=\mathbf{0}\left(\right.$ since $\left.\mathcal{W}_{3, \mathbf{n}-2}=\mathbf{0}\right)$.

The solvability conditions for (61) can be expressed as

$$
\left(\begin{array}{cc}
2 & i \mathcal{S}_{3, n} \\
2 \gamma_{0} & 2\left(\lambda_{3}+i \mu_{3} c_{g-1}\left(\mu_{0}+m\right)\right)
\end{array}\right)\left(\begin{array}{l}
\lambda_{4} \\
\gamma_{1}
\end{array}\right)+i \gamma_{2}\left(\begin{array}{c}
0 \\
\mathcal{T}_{4, m}
\end{array}\right)=-2 i\left(\begin{array}{c}
\mu_{4} c_{g_{1}}\left(\mu_{0}+n\right)-\mathcal{P}_{4, n} \\
\gamma_{0}\left(\mu_{4} c_{g_{-1}}\left(\mu_{0}+m\right)-\mathcal{P}_{4, m}\right)
\end{array}\right) .
$$


Expressions for $\mathcal{P}_{4, j}$ are in the file in Data Availability Statement. Using the solvability condition (A22b) together with the collision condition (24) shows that $\mathcal{T}_{4, m} \equiv 0$. What remains is a linear system for $\lambda_{4}$ and $\gamma_{1}$.

If $\alpha \neq 1.1862 \ldots$, then an application of the third-order solvability condition (A28a) shows that, for $\mu_{3} \in\left(-M_{3}, M_{3}\right)$,

$$
\operatorname{det}\left(\begin{array}{cc}
2 & i \mathcal{S}_{3, n} \\
2 \gamma_{0} & 2\left(\lambda_{3}+i \mu_{3} c_{g_{-1}}\left(\mu_{0}+m\right)\right)
\end{array}\right)=8 \lambda_{3, r},
$$

where $\lambda_{3, r}=\operatorname{Re}\left(\lambda_{3}\right)$. For $\mu_{3}$ in this interval, $\lambda_{3, r} \neq 0$ by construction; thus, (62) is an invertible linear system.

We solve (62) for $\lambda_{4}$ by Cramer's rule, using (A28a) to eliminate the dependence on $\gamma_{0}$ and $\mathcal{S}_{3, n}$. Then,

$$
\begin{aligned}
\lambda_{4}=-i & {\left[\frac{\left(\lambda_{3}+i \mu_{3} c_{g_{-1}}\left(\mu_{0}+m\right)\right)\left(\mu_{4} c_{g_{1}}\left(\mu_{0}+n\right)-\mathcal{P}_{4, n}\right)}{2 \lambda_{3, r}}\right.} \\
& \left.+\frac{\left(\lambda_{3}+i \mu_{3} c_{g_{1}}\left(\mu_{0}+n\right)\right)\left(\mu_{4} c_{g_{-1}}\left(\mu_{0}+m\right)-\mathcal{P}_{4, m}\right)}{2 \lambda_{3, r}}\right] .
\end{aligned}
$$

We decompose (64) into its real and imaginay components, noting $\mathcal{P}_{4, n}, \mathcal{P}_{4, m}$, and $\mu_{4} \in \mathbb{R}$. We find $\lambda_{4}=\lambda_{4, r}+i \lambda_{4, i}$, where

$$
\begin{aligned}
\lambda_{4, r} & =\frac{\mu_{3}\left(c_{g_{-1}}\left(\mu_{0}+m\right)-c_{g_{1}}\left(\mu_{0}+n\right)\right)\left[-\mu_{4}\left(c_{g_{-1}}\left(\mu_{0}+m\right)-c_{g_{1}}\left(\mu_{0}+n\right)\right)+\mathcal{P}_{4, m}-\mathcal{P}_{4, n}\right]}{4 \lambda_{3, r}}, \\
\lambda_{4, i} & =-\frac{1}{2}\left[\mu_{4}\left(c_{g_{-1}}\left(\mu_{0}+m\right)+c_{g_{1}}\left(\mu_{0}+n\right)\right)-\left(\mathcal{P}_{4, m}+\mathcal{P}_{4, n}\right)\right] .
\end{aligned}
$$

As $\left|\mu_{3}\right| \rightarrow M_{3}, \lambda_{3, r} \rightarrow 0$. If $\lambda_{4, r}$ is to remain bounded, the numerator of (65a) must vanish in this limit. Since $c_{g_{-1}}\left(\mu_{0}+m\right) \neq c_{g_{1}}\left(\mu_{0}+n\right)$, we must have

$$
\mu_{4}=\frac{\mathcal{P}_{4, m}-\mathcal{P}_{4, n}}{c_{g_{-1}}\left(\mu_{0}+m\right)-c_{g_{1}}\left(\mu_{0}+n\right)} .
$$

We refer to this equality as the regular curve condition: it ensures that the curve asymptotic to the $p=3$ isola remains continuous near its intersections with the imaginary axis. From the regular curve condition, we obtain

$$
\lambda_{4}=-i\left(\frac{\mathcal{P}_{4, m} c_{g_{1}}\left(\mu_{0}+n\right)-\mathcal{P}_{4, n} c_{g_{-1}}\left(\mu_{0}+m\right)}{c_{g_{-1}}\left(\mu_{0}+m\right)-c_{g_{1}}\left(\mu_{0}+n\right)}\right) .
$$

As expected, the Floquet parameterization and imaginary component of the $p=3$ isola have a nonzero correction at $\mathcal{O}\left(\varepsilon^{4}\right)$. These corrections improve the agreement between numerical and asymptotic results observed at the previous order (Figures 9 and 10). No corrections to the real component of the isola are found at fourth order.

Remark 8. If $\alpha=1.1862 \ldots$, one can show that $\lambda_{3}=0=\mu_{3}$ and $\mathcal{S}_{3, n}=0$. Applying the Fredholm alternative to (62) gives (66). Then, $\lambda_{4}$ is given by (67), and $\gamma_{0}=1$. The constant $\gamma_{1}$ remains arbitrary at this order for this value of $\alpha$ only. 

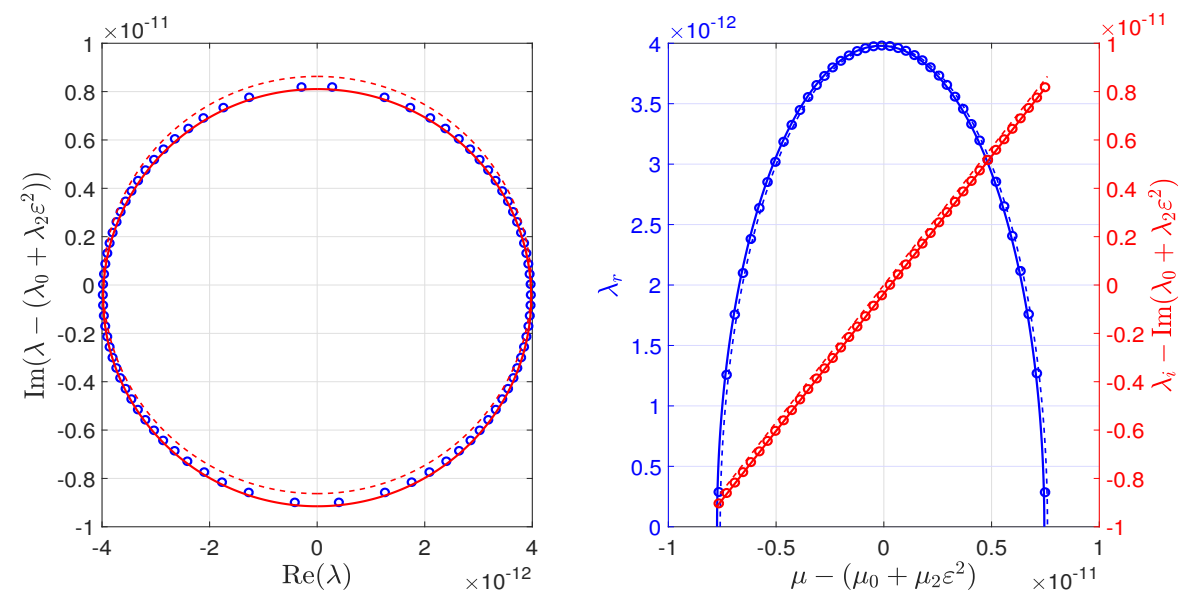

Figure 9. (Left) The $p=3$ isola with $\alpha=1$ and $\varepsilon=5 \times 10^{-4}$. The zeroth- and second-order imaginary corrections are removed for better visibility. Solid and dashed red curves are given by perturbation calculations to $\mathcal{O}\left(\varepsilon^{4}\right)$ and $\mathcal{O}\left(\varepsilon^{3}\right)$, respectively. Blue circles are a subset of spectral elements from the numerically computed isola using FFH. (Right) The Floquet parameterization of the real (blue) and imaginary (red) components of the isola. The zeroth- and second-order corrections of both the imaginary component and Floquet exponent are removed for better visibility. Solid and dashed curves illustrate perturbation calculations to $\mathcal{O}\left(\varepsilon^{4}\right)$ and $\mathcal{O}\left(\varepsilon^{3}\right)$, respectively. Circles indicate FFH results.
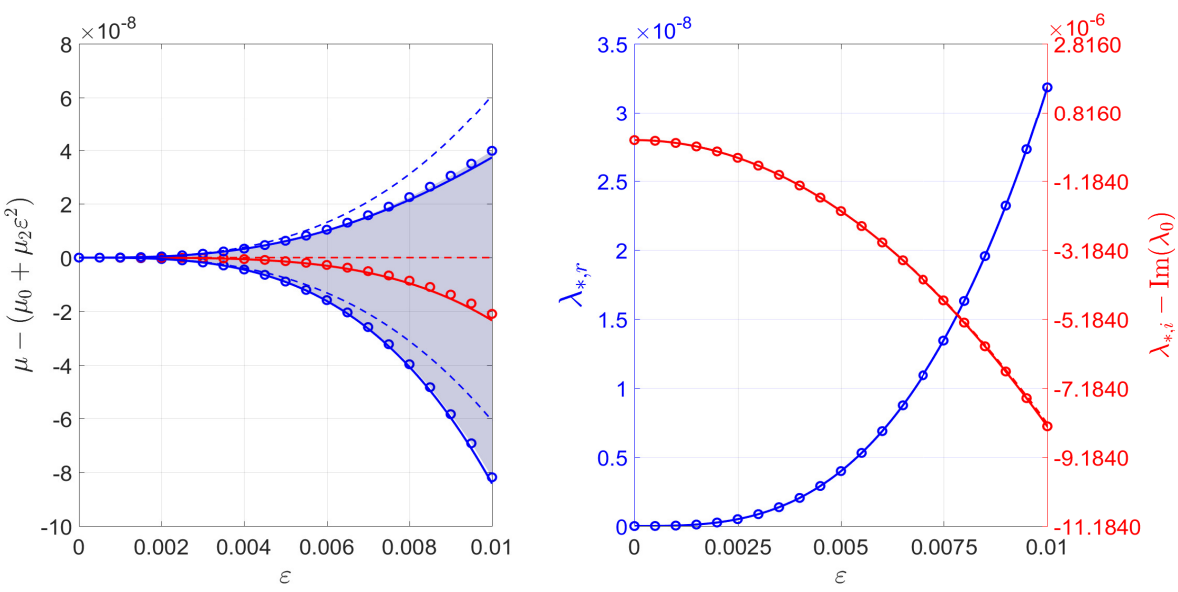

Figure 10. (Left)The interval of Floquet exponents that parameterize the $p=3$ isola as a function of $\varepsilon$ for $\alpha=1$. The zeroth- and second-order Floquet corrections are removed for better visibility. Solid and dashed blue curves indicate the boundaries of this interval according to perturbation calculations to $\mathcal{O}\left(\varepsilon^{4}\right)$ and $\mathcal{O}\left(\varepsilon^{3}\right)$, respectively. Blue circles indicate the boundaries computed numerically by FFH. The solid red curve gives the Floquet exponent corresponding to the most unstable spectral element of the isola according to our perturbation calculations. Red circles indicate the same but computed numerically using FFH. (Right) The real (blue) and imaginary (red) components of the most unstable spectral element of the isola as a function of $\varepsilon$. The zeroth-order imaginary correction is removed for better visibility. Solid and dashed curves illustrate perturbation calculations to $\mathcal{O}\left(\varepsilon^{4}\right)$ and $\mathcal{O}\left(\varepsilon^{3}\right)$, respectively. Circles illustrate FFH results.

\section{Conclusions}

We have extended a formal perturbation method, introduced in [22], to obtain asymptotic behavior of the largest ( $p=2,3$ ) high-frequency instabilities of small-amplitude, HPT-BW Stokes waves. In particular, we have computed explicit expressions for (i) the interval of Floquet exponents that asymptotically parameterize the pth isola, (ii) the leading-order behavior of its most unstable spectral elements, (iii) the leading-order curve 
asymptotic to the isola, and (iv) wavenumbers that do not have a pth isola. Items (i)-(iii) can be extended to higher-order if necessary using the regular curve condition. In all instances, our perturbation calculations are in excellent agreement with numerical results computed by the FFH method [12].

We restrict to $p=2$ and $p=3$ in this work, but our method can provide asymptotic expressions for $p>3$ isolas. We conjecture that this method yields the first real-component correction of the isola at $\mathcal{O}\left(\varepsilon^{p}\right)$, similar to the cases $p=2$ and $p=3$. If correct, this conjecture highlights the main difficulty of computing higher-order high-frequency instabilities, both numerically and perturbatively.

The asymptotic expressions derived in this paper are intimidating and cumbersome. Although it is satisfying to have asymptotic expressions for the results previously obtained only numerically, this is not the main point of our work. Rather, (i) the perturbation method demonstrated allows one to approximate an entire isola at once, going beyond standard eigenvalue perturbation theory [27], (ii) the results obtained constitute a first step toward a proof of the presence of the high-frequency instabilities, and (iii) the asymptotic expressions for the range of Floquet exponents allow for a far more efficient numerical computation of the high-frequency isolas, which are difficult to track numerically as the amplitude of the solution increases.

Author Contributions: Conceptualization, B.D.; formal analysis, R.C.; supervision, B.D. and O.T.; writing — original draft preparation, R.C.; writing—review and editing, B.D. and O.T. All authors have read and agreed to the published version of the manuscript.

Funding: This work was partially funded by the ARCS (Achievement Rewards for College Scientists) Foundation Fellowship.

Institutional Review Board Statement: Not applicable.

Informed Consent Statement: Not applicable.

Data Availability Statement: The asymptotic expressions derived in this manuscript may be found at https://zenodo.org/record/4637882.

Conflicts of Interest: The authors declare no conflict of interest.

\section{Abbreviations}

The following abbreviations are used in this manuscript:

HPT-BW Hur-Pandey-Tao-Boussinesq-Whitham System

FFH Floquet-Fourier-Hill Method

WWP Water Wave Problem (in 1D)

\section{Appendix A. Stokes Wave Expansions}

Below are the Stokes wave expansions of (11) (with $\mathcal{I}_{j}=0$ ) to the fourth order in the small-amplitude parameter $\varepsilon$. In what follows,

$$
C_{k}^{2}=\frac{\tanh (\alpha k)}{\alpha k} \quad \text { and } \quad D_{z}=\frac{1}{c_{0}^{2}-z^{2}}, \quad \text { where } \quad c_{0}^{2}=C_{1}^{2} .
$$

For the surface displacement $\eta_{S}(x ; \varepsilon)$,

$$
\begin{aligned}
\eta_{S}(x ; \varepsilon)= & \varepsilon \eta_{1}(x)+\varepsilon^{2} \eta_{2}(x)+\varepsilon^{3} \eta_{3}(x)+\varepsilon^{4} \eta_{4}(x)+\mathcal{O}\left(\varepsilon^{5}\right) \\
=\varepsilon & \varepsilon \cos (x)+\left(N_{2,0}+2 N_{2,2} \cos (2 x)\right) \varepsilon^{2}+2 N_{3,3} \varepsilon^{3} \cos (3 x) \\
& +\left(N_{4,0}+2 N_{4,2} \cos (2 x)+2 N_{4,4} \cos (4 x)\right) \varepsilon^{4}+\mathcal{O}\left(\varepsilon^{5}\right),
\end{aligned}
$$


with

$$
\begin{aligned}
N_{2,0}= & \frac{3 c_{0}^{2} D_{1}}{4}, \\
N_{2,2}= & \frac{3 c_{0}^{2} D_{C_{2}}}{8}, \\
N_{3,3}= & \frac{c_{0}^{2} D_{C_{2}} D_{C_{3}}}{16}\left(5 c_{0}^{2}+4 C_{2}^{2}\right) \\
N_{4,0}= & -\frac{3 c_{0}^{2} D_{1}^{3} D_{C_{2}}^{2}}{64}\left(25 c_{0}^{8}+4 C_{2}^{4}+2 c_{0}^{2} C_{2}^{2}\left(-7+2 C_{2}^{2}\right)-4 c_{0}^{6}\left(2+11 C_{2}^{2}\right)\right. \\
& \left.\quad+c_{0}^{4}\left(1+22 C_{2}^{2}+10 C_{2}^{4}\right)\right) \\
N_{4,2}= & \frac{c_{0}^{2} D_{1} D_{C_{2}}^{3} D_{C_{3}}}{64}\left(-20 c_{0}^{8}+4 C_{2}^{4} C_{3}^{2}+c_{0}^{6}\left(2+31 C_{2}^{2}+50 C_{3}^{2}\right)\right. \\
& \left.\quad+c_{0}^{2} C_{2}^{2}\left(C_{3}^{2}+2 C_{2}^{2}\left(10+7 C_{3}^{2}\right)\right)-c_{0}^{4}\left(38 C_{2}^{4}+32 C_{3}^{2}+C_{2}^{2}\left(-5+37 C_{3}^{2}\right)\right)\right) \\
N_{4,4}= & \frac{c_{0}^{2} D_{C_{2}}^{2} D_{C_{3}} D_{C_{4}}}{128}\left(35 c_{0}^{6}-20 C_{2}^{4} C_{3}^{2}+5 c_{0}^{4}\left(4 C_{2}^{2}+5 C_{3}^{2}\right)-4 c_{0}^{2}\left(7 C_{2}^{4}+8 C_{2}^{2} C_{3}^{2}\right)\right) .
\end{aligned}
$$

For the horizontal velocity $u_{S}(x ; \varepsilon)$ along $\eta_{S}(x ; \varepsilon)$,

$$
\begin{aligned}
u_{S}(x ; \varepsilon)= & \varepsilon u_{1}(x)+\varepsilon^{2} u_{2}(x)+\varepsilon^{3} u_{3}(x)+\varepsilon^{4} u_{4}(x)+\mathcal{O}\left(\varepsilon^{5}\right) \\
= & c_{0} \varepsilon \cos (x)+\left(U_{2,0}+2 U_{2,2} \cos (2 x)\right) \varepsilon^{2}+\left(2 U_{3,1} \cos (x)+2 U_{3,3} \cos (3 x)\right) \varepsilon^{3} \\
& +\left(U_{4,0}+2 U_{4,2} \cos (2 x)+2 U_{4,4} \cos (4 x)\right) \varepsilon^{4}+\mathcal{O}\left(\varepsilon^{5}\right)
\end{aligned}
$$

with

$$
\begin{aligned}
& U_{2,0}=\frac{c_{0} D_{1}}{4}\left(2+c_{0}^{2}\right), \\
& U_{2,2}=\frac{c_{0} D_{C_{2}}}{8}\left(2 C_{2}^{2}+c_{0}^{2}\right), \\
& U_{3,1}=\frac{3 c_{0}^{3} D_{1} D_{C_{2}}}{32}\left(1-3 c_{0}^{2}+2 C_{2}^{2}\right), \\
& U_{3,3}=\frac{c_{0} D_{C_{2}} D_{C_{3}}}{16}\left(c_{0}^{4}+2 C_{2}^{2} C_{3}^{2}+2 c_{0}^{2}\left(C_{2}^{2}+2 C_{3}^{2}\right)\right), \\
& U_{4,0}=\frac{-3 c_{0}^{3} D_{1}^{3} D_{C_{2}}^{2}\left(2+c_{0}^{2}\right)}{64}\left(2 c_{0}^{4}\left(-2+5 c_{0}^{2}\right)+C_{2}^{2}\left(-3+8 c_{0}^{2}-17 c_{0}^{4}\right)+2 C_{2}^{4}\left(1+2 c_{0}^{2}\right)\right), \\
& U_{4,2}=\frac{c_{0} D_{1} D_{C_{2}}^{3} D_{C_{3}}}{128}\left(-25 c_{0}^{10}+8 C_{2}^{6} C_{3}^{2}+c_{0}^{8}\left(7-C_{2}^{2}+45 C_{3}^{2}\right)+c_{0}^{6}\left(C_{2}^{2}+32 C_{2}^{4}\right.\right. \\
& \left.\left.\quad+C_{3}^{2}\left(-27+37 C_{2}^{2}\right)\right)-c_{0}^{4}\left(60 C_{2}^{6}+37 C_{2}^{2} C_{3}^{2}+C_{2}^{4}\left(-22+56 C_{3}^{2}\right)\right)\right), \\
& U_{4,4}=\frac{c_{0} D_{C_{2}}^{2} D_{C_{3}} D_{C_{4}}}{128}\left(5 c_{0}^{8}-8 C_{2}^{4} C_{3}^{2} C_{4}^{2}+2 c_{0}^{4}\left(-2 C_{2}^{4}+5 C_{3}^{2} C_{4}^{2}+6 C_{2}^{2}\left(-C_{3}^{2}+C_{4}^{2}\right)\right)\right. \\
& \left.\quad+c_{0}^{6}\left(8 C_{2}^{2}+15\left(C_{3}^{2}+2 C_{4}^{2}\right)\right)-4 c_{0}^{2}\left(5 C_{2}^{2} C_{3}^{2} C_{4}^{2}+3 C_{2}^{4}\left(C_{3}^{2}+2 C_{4}^{2}\right)\right)\right) .
\end{aligned}
$$

For the velocity of the Stokes waves $c(\varepsilon)$,

$$
c(\varepsilon)=c_{0}+c_{2} \varepsilon^{2}+c_{4} \varepsilon^{4}+\mathcal{O}\left(\varepsilon^{6}\right),
$$

with 


$$
\begin{aligned}
c_{2}= & \frac{3 c_{0} D_{1} D_{C_{2}}}{16}\left(c_{0}^{2}+5 c_{0}^{4}-2 C_{2}^{2}\left(2+c_{0}^{2}\right)\right), \\
c_{4}= & \frac{3 c_{0} D_{1}^{3} D_{C_{2}}^{3} D_{C_{3}}}{512}\left(3 c _ { 0 } ^ { 2 } \left(c_{0}^{6}-3 c_{0}^{8}+15 c_{0}^{10}-85 c_{0}^{12}+c_{0}^{4} C_{2}^{2}\left(11+3 c_{0}^{2}-3 c_{0}^{4}+205 c_{0}^{6}\right)\right.\right. \\
& \left.\quad-4 c_{0}^{2} C_{2}^{4}\left(-2+15 c_{0}^{2}+3 c_{0}^{4}+38 c_{0}^{6}\right)-4 C_{2}^{6}\left(-4+12 c_{0}^{2}-27 c_{0}^{4}+c_{0}^{6}\right)\right) \\
& +C_{3}^{2}\left(c_{0}^{6}\left(-103+309 c_{0}^{2}-345 c_{0}^{4}+355 c_{0}^{6}\right)-3 c_{0}^{4} C_{2}^{2}\left(31-57 c_{0}^{2}+57 c_{0}^{4}+185 c_{0}^{6}\right)\right. \\
& \left.\left.+36 c_{0}^{2} C_{2}^{4}\left(2-3 c_{0}^{2}+9 c_{0}^{4}+10 c_{0}^{6}\right)-4 C_{2}^{6}\left(2+c_{0}^{2}\right)\left(-2+7 c_{0}^{2}+13 c_{0}^{4}\right)\right)\right) .
\end{aligned}
$$

\section{Appendix B. Collision Condition}

Up to relabeling $m$ and $n$, (24) simplifies to

$$
\Omega_{1}\left(\mu_{0}+n\right)=\Omega_{-1}\left(\mu_{0}+m\right) \neq 0 .
$$

With $k=\mu_{0}+n$ and $p=m-n$, (A8) becomes

$$
\Omega_{1}(k)=\Omega_{-1}(k+p) \neq 0 .
$$

We refer to (A9) as the collision condition. We prove that, for each $p \in \mathbb{Z} \backslash\{0, \pm 1\}$, there exists a unique $k(p ; \alpha)$ that satisfies the collision condition. These solutions $k(p ; \alpha)$ are distinct from each other (for each $\alpha>0$ ) and result in an infinite number of distinct collision points on the imaginary axis, according to (24). First, we establish important monotonicity properties of $\Omega_{\sigma}(k)$, defined in (22).

Lemma A1. The function $\omega_{\alpha}(k)=\operatorname{sgn}(k) \sqrt{\alpha k \tanh (\alpha k)}$ is strictly increasing for $k \in \mathbb{R}$. If $|k|>1$, then $\omega_{\alpha}^{\prime}(k)<\alpha\left|c_{0}\right|$, where $c_{0}^{2}=\tanh (\alpha) / \alpha$.

Proof. A direct calculation shows

$$
\omega_{\alpha}^{\prime}(k)=\frac{1}{2}\left(\sqrt{\frac{\alpha \tanh (\alpha k)}{k}}+\alpha \sqrt{\frac{\alpha k}{\sinh (\alpha k)}} \operatorname{sech}^{3 / 2}(\alpha k)\right),
$$

from which $\omega_{\alpha}^{\prime}(k)>0$. This proves the first claim. Since $\tanh (\alpha k) /(\alpha k) \leq 1, \alpha k / \sinh (\alpha k) \leq 1$, and $\operatorname{sech}(\alpha k) \leq 1$, (A10) gives

$$
\omega_{\alpha}^{\prime}(k) \leq \frac{1}{2}\left(\sqrt{\frac{\alpha \tanh (\alpha k)}{k}}+\alpha \operatorname{sech}(\alpha k)\right) .
$$

Since $\alpha>0, \sinh (\alpha) / \alpha>1>\operatorname{sech}(\alpha)$, so that $\operatorname{sech}(\alpha)<\left|c_{0}\right|$. Because $\operatorname{sech}(z)$ is even and strictly decreasing for $z>0$, we have

$$
\operatorname{sech}(\alpha k)<\left|c_{0}\right|, \text { for }|k|>1 .
$$

Similarly, $\operatorname{since} \tanh (z) / z$ is even and strictly decreasing for $z>0$,

$$
\sqrt{\frac{\alpha \tanh (\alpha k)}{k}}<\alpha \sqrt{\frac{\tanh (\alpha)}{\alpha}}=\alpha\left|c_{0}\right|, \text { for }|k|>1 .
$$

Together with (A11), inequalities (A12) and (A13) imply $\omega_{\alpha}^{\prime}(k)<\alpha\left|c_{0}\right|$ for $|k|>1$.

Lemma A2. If $c_{0}>0, \Omega_{-1}(k)$ is strictly decreasing for $k \in \mathbb{R}$, and $\Omega_{1}(k)$ is strictly decreasing for $|k|>1$. If $c_{0}<0, \Omega_{1}(k)$ is strictly increasing for $k \in \mathbb{R}$, and $\Omega_{-1}(k)$ is strictly increasing for $|k|>1$. 
Proof. Suppose $c_{0}>0$. By definition, $\Omega_{\sigma}^{\prime}(k)=-\alpha c_{0}+\sigma \omega_{\alpha}^{\prime}(k)$. If $\sigma=-1$, we use $\omega_{\alpha}^{\prime}(k)>0$ from Lemma A1 to conclude $\Omega_{-1}^{\prime}(k)<0$. If $\sigma=1$ and $|k|>1$, we use $\omega_{\alpha}^{\prime}(k)<\alpha\left|c_{0}\right|$ from Lemma A1 to conclude $\Omega_{1}^{\prime}(k)=-\alpha c_{0}+\omega_{\alpha}^{\prime}(k)<0$, since $c_{0}>0$. An analogous proof holds when $c_{0}<0$.

In what follows, we consider $c_{0}>0$, which corresponds to right-traveling Stokes waves. Similar statements hold when $c_{0}<0$ if one rewrites the collision condition (A9) as $\Omega_{-1}(k)=\Omega_{1}(k+p) \neq 0$, where $k$ and $p$ are redefined appropriately.

Lemma A3. For each $p \in \mathbb{R}$ and $\alpha>0$, there exists a unique $k(p ; \alpha) \in \mathbb{R}$ such that $\Omega_{1}(k(p ; \alpha))=$ $\Omega_{-1}(k(p ; \alpha)+p)$. If $p \in \mathbb{Z}$ and $c_{0}>0$, we have $\cdots<k(1 ; \alpha)<k(0 ; \alpha)<k(-1 ; \alpha)<\cdots$. Moreover, $|k(p ; \alpha)|>|p|$ for $p \in \mathbb{Z} \backslash\{0, \pm 1\}$ and $c_{0}>0$.

Proof. Fix $p \in \mathbb{R}$ and $\alpha>0$. Define $F(k, p)=\Omega_{1}(k)-\Omega_{-1}(k+p)$. Then,

$$
F(k, p) \sim 2 k \sqrt{\frac{\alpha}{|k|}}+\mathcal{O}\left(\frac{1}{\sqrt{|k|}}\right) \quad \text { as } \quad|k| \rightarrow \infty .
$$

Since $F$ has opposite signs as $k \rightarrow \pm \infty$, there exists at least one root, denoted $k(p ; \alpha)$. Since $\partial_{k} F(k, p)=\omega^{\prime}(k)+\omega^{\prime}(k+p)>0$ by Lemma A1, $k(p ; \alpha)$ is the only $\operatorname{root}$ of $F$ in $\mathbb{R}$, proving the first claim of the theorem.

To prove the second claim, we differentiate $F(k(p ; \alpha), p)$ with respect to $p$. Using the definition of $\Omega_{\sigma}$,

$$
k^{\prime}(p)=\frac{\Omega_{-1}^{\prime}(k(p ; \alpha)+p)}{\omega^{\prime}(k(p ; \alpha))+\omega^{\prime}(k(p ; \alpha)+p)},
$$

which is well-defined since $\partial_{k} F(k, p)>0$. If $c_{0}>0$, then Lemma A2 implies $k^{\prime}(p)<0$. If $p$ is restricted to $\mathbb{Z}$, we have $\cdots<k(1 ; \alpha)<k(0 ; \alpha)<k(-1 ; \alpha)<\cdots$, as desired.

To prove the third claim, we first consider $p>1$. Suppose $k(p ; \alpha) \geq-p$. Since $\omega_{\alpha}(k)$ is odd and strictly increasing by Lemma A1,

$$
\begin{aligned}
\omega_{\alpha}(k(p ; \alpha)) & \geq-\omega_{\alpha}(p), \\
\omega_{\alpha}(k(p ; \alpha)+p) & \geq \omega_{\alpha}(0)=0 .
\end{aligned}
$$

Using the definition of $\Omega_{\sigma}, F(k(p ; \alpha), p)=0$ can be rewritten as

$$
\omega_{\alpha}(k(p ; \alpha))+\omega_{\alpha}(k(p ; \alpha)+p)=-\alpha c_{0} p .
$$

Together with (A17), inequalities (A16a) and (A16b) imply

$$
-\omega_{\alpha}(p) \leq-\alpha c_{0} p \quad \Rightarrow \quad \frac{\omega_{\alpha}(p)}{p} \geq \alpha c_{0}=\frac{\omega_{\alpha}(1)}{1},
$$

a contradiction, since $\omega_{\alpha}(z) / z$ is strictly decreasing for $z>0$. Therefore, $k(p ; \alpha)<-p$ for $p>1$. Since $\Omega_{\sigma}(k)$ is odd, $k(p ; \alpha)=-k(-p ; \alpha)$. Therefore, when $p<-1, k(p ; \alpha)>-p$. Combining the two cases yields $|k(p ; \alpha)|>|p|$ whenever $p \in \mathbb{Z} \backslash\{0, \pm 1\}$ and $c_{0}>0$, as desired.

Lemma $\mathrm{A} 3$ has several consequences:

1. When $c_{0}>0, k(p ; \alpha)<0$ for $p>0$, and $k(p ; \alpha)>0$ for $p<0$.

2. When $c_{0}>0, k(p ; \alpha) \rightarrow \pm \infty$ as $p \rightarrow \mp \infty$. In fact, the sequence $\{k(p ; \alpha)\}$ must grow at least linearly as $|p| \rightarrow \infty$. Formal arguments suggest quadratic growth in this limit.

3. The products $k(p ; \alpha)(k(p ; \alpha)+p)>0$ and $\omega_{\alpha}(k(p ; \alpha)) \omega_{\alpha}(k(p ; \alpha)+p)>0$ when $c_{0}>0$. The latter of these products is related to the Krein signature condition proposed 
in [26]. In effect, Lemma A3 provides a different proof that collided eigenvalues (24) have opposite Krein signatures, consistent with [3].

The above results lead to the following theorem.

Theorem A1. Let $c_{0}>0$. If $p \in\{0, \pm 1\}$, then the collision condition (A9) is not satisfied. If $p \in \mathbb{Z} \backslash\{0, \pm 1\}$, then $k(p ; \alpha)$ solves the collision condition. Moreover, $\cdots<\lambda_{i, 3}<\lambda_{i, 2}<$ $0<\lambda_{i,-2}<\lambda_{i,-3}<\cdots$, where $\lambda_{i, p}$ is the imaginary part of the collision point corresponding to $k(p ; \alpha)$.

Proof. When $p=0$ or \pm 1 , we have $k(p ; \alpha)=0$ or $\mp 1$, respectively, by inspection. It follows that $\Omega_{1}(k(p ; \alpha))=0$ in all three cases, so (A9) is not satisfied. This proves the first claim.

To prove the second claim, consider the sequence $\left\{\Omega_{1}(k(p ; \alpha))\right\}, p \in \mathbb{Z} \backslash\{0, \pm 1\}$. From Lemma A3, $\{k(p ; \alpha)\}$ is a strictly decreasing sequence, and each element of this sequence satisfies $|k(p ; \alpha)|>|p|>1$. Thus, Lemma A2 holds, and the sequence $\left\{\Omega_{1}(k(p ; \alpha))\right\}$ is strictly increasing. Since $\Omega_{1}( \pm 1)=0$, we have $\Omega_{1}(k(p ; \alpha)) \neq 0$. This proves that $k(p ; \alpha)$ satisfies the collision condition (A9) for the relevant values of $p$.

The proof of the third claim is immediate since $\left\{\Omega_{1}(k(p ; \alpha))\right\}$ is strictly increasing.

Let $\mu_{0}=k(p ; \alpha)-[k(p ; \alpha)]$ for $p \in \mathbb{Z} \backslash\{0, \pm 1\}$, where [.] denotes the nearest integer function. Then, $\mu_{0}$ is the unique Floquet exponent in $[-1 / 2,1 / 2]$ for which $\lambda_{0, \mu_{0}, n}^{(1)}$ and $\lambda_{0, \mu_{0}, m}^{(-1)}$ satisfy (24) with $n=[k(p ; \alpha)]$ and $m=n+p$.

\section{Appendix C. Detailed Calculations of the $p=3$ Instability}

Appendix C.1. The $\mathcal{O}(\varepsilon)$ Problem

Substituting expansions (13), (26), and (30) into the spectral problem (19), equating terms of $\mathcal{O}(\varepsilon)$, and using expression for $\eta_{1}, u_{1}$, and $\mathbf{w}_{0}$ to simplify, we find

$$
\left(\mathcal{L}_{0, \mu_{0}}-\lambda_{0}\right) \mathbf{w}_{\mathbf{1}}=\sum_{j=n-1}^{m+1} \mathbf{T}_{\mathbf{1}, \mathrm{j}} e^{i j x} .
$$

Expressions for $\mathbf{T}_{\mathbf{1}, \mathrm{j}}$ depend on $\mu_{0}, \alpha$, and $\gamma_{0}$; see the file in Data Availability Statement. Since $m-n=3, j \in\{n-1, n, 1+n, m-1, m, m+1\}$. The functional expressions for $\mathbf{T}_{\mathbf{1 , n - 1}}$ and $\mathbf{T}_{\mathbf{1}, \mathbf{m}+\mathbf{1}}$ are identical to those in the $p=2$ case (they do not evaluate to the same vectors, however, as $\mu_{0}$ is different for $p=2$ and $p=3$ in general).

Solvability conditions for (A19) simplify to $\mu_{1}=0=\lambda_{1}$. Together with the normalization (28), these conditions guarantee a solution to (A19) of the form

$$
\mathbf{w}_{\mathbf{1}}=\sum_{\substack{j=n-1 \\
j \neq n, m}}^{m+1} \mathcal{W}_{\mathbf{1}, \mathrm{j}} \mathrm{e}^{i j x}+\gamma_{1}\left(\begin{array}{c}
1 \\
-\frac{\omega_{\alpha}\left(m+\mu_{0}\right)}{\alpha\left(m+\mu_{0}\right)}
\end{array}\right) e^{i m x},
$$

where $\gamma_{1}$ is arbitrary and expressions for $\mathcal{W}_{1, j}$ are found in the file in Data Availability Statement. Because $\mathbf{T}_{\mathbf{1 , n}-\mathbf{1}}$ and $\mathbf{T}_{\mathbf{1 , \mathbf { m } + \mathbf { 1 }}}$ are identical to their $p=2$ counterparts, $\mathcal{W}_{\mathbf{1 , n}-\mathbf{1}}$ and $\mathcal{W}_{\mathbf{1 , m}+\mathbf{1}}$ are as well.

Appendix C.2. The $\mathcal{O}\left(\varepsilon^{2}\right)$ Problem

The $\mathcal{O}\left(\varepsilon^{2}\right)$ problem takes the same form as (41). Evaluating at $\eta_{j}, u_{j}$, and $\mathbf{w}_{\mathbf{j}-\mathbf{1}}$ for $j \in\{1,2\}$, we find

$$
\left(\mathcal{L}_{0, \mu_{0}}-\lambda_{0}\right) \mathbf{w}_{\mathbf{2}}=\sum_{\substack{j=n-2 \\ j \neq n-1}}^{m+2} \mathbf{T}_{2, j} \mathrm{e}^{i j x}
$$




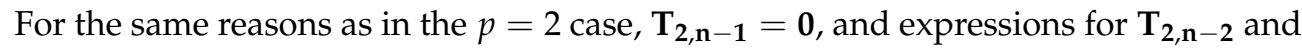
$\mathbf{T}_{2, \mathbf{m}+\mathbf{2}}$ are identical to their $p=2$ counterparts.

Since $\gamma_{0} \neq 0$, the solvability conditions for (A21) simplify to

$$
\begin{aligned}
\lambda_{2}+i \mu_{2} c_{g_{1}}\left(\mu_{0}+n\right)-i \mathcal{P}_{2, n} & =0, \\
\lambda_{2}+i \mu_{2} c_{g_{-1}}\left(\mu_{0}+m\right)-i \mathcal{P}_{2, m} & =0,
\end{aligned}
$$

where $\mathcal{P}_{2, j}$ are independent of $\lambda_{2}, \mu_{2}, \gamma_{0}$, and $\gamma_{1}$; see the file in Data Availability Statement. Note that these terms are distinct from those introduced in (45).

Solving (A22a) and (A22b) for $\lambda_{2}$ and $\mu_{2}$ yields

$$
\begin{aligned}
& \lambda_{2}=-i\left(\frac{\mathcal{P}_{2, m} c_{g_{1}}\left(\mu_{0}+n\right)-\mathcal{P}_{2, n} c_{g_{-1}}\left(\mu_{0}+m\right)}{c_{g_{-1}}\left(\mu_{0}+m\right)-c_{g_{1}}\left(\mu_{0}+n\right)}\right), \\
& \mu_{2}=\frac{\mathcal{P}_{2, m}-\mathcal{P}_{2, n}}{c_{g_{-1}}\left(\mu_{0}+m\right)-c_{g_{1}}\left(\mu_{0}+n\right)} .
\end{aligned}
$$

Thus, the spectral elements and Floquet parameterization of the $p=3$ isola have nontrivial corrections at $\mathcal{O}\left(\varepsilon^{2}\right)$. However, since $\operatorname{Re}\left(\lambda_{2}\right)=0$, we have yet to determine the leading-order behavior of the isola. We find this at the next order.

Imposing solvability conditions (A22a) and (A22b) as well as the normalization condition on $\mathbf{w}_{\mathbf{2}}$, the solution of (A21) is

$$
\mathbf{w}_{\mathbf{2}}=\sum_{\substack{j=n-2 \\
j \neq n-1}}^{m+1} \mathcal{W}_{2, j} \mathrm{j}^{i j x}+\gamma_{2}\left(\begin{array}{c}
1 \\
-\frac{\omega_{\alpha}\left(m+\mu_{0}\right)}{\alpha\left(m+\mu_{0}\right)}
\end{array}\right) e^{i m x},
$$

where $\gamma_{2}$ is an arbitrary constant. Since $\mathbf{T}_{\mathbf{2}, \mathbf{n}-\mathbf{1}}=\mathbf{0}, \mathcal{W}_{\mathbf{2 , \mathbf { n } - \mathbf { 1 }}}=\mathbf{0}$.

Appendix C.3. The $\mathcal{O}\left(\varepsilon^{3}\right)$ Problem

At $\mathcal{O}\left(\varepsilon^{3}\right)$, the spectral problem (19) takes the form

$$
\left(\mathcal{L}_{0, \mu_{0}}-\lambda_{0}\right) \mathbf{w}_{\mathbf{3}}=\sum_{j=2}^{3} \lambda_{j} \mathbf{w}_{\mathbf{3}-\mathbf{j}}-\left.\sum_{j=1}^{3} \mathcal{L}_{j}\right|_{\mu_{1}=0} \mathbf{w}_{3-\mathbf{j}},
$$

where $\left.\mathcal{L}_{j}\right|_{\mu_{1}=0}$ for $j \in\{1,2\}$ are as before and

$$
\left.\mathcal{L}_{3}\right|_{\mu_{1}=0}=\alpha\left(\begin{array}{cc}
-u_{3}^{\prime}-i \mu_{2} u_{1}-u_{3}\left(i \mu_{0}+\partial_{x}\right)+i \mu_{3} c_{0} & -\eta_{3}^{\prime}-i \mu_{3}-i \eta_{1} \mu_{2}-\eta_{3}\left(i \mu_{0}+\partial_{x}\right) \\
-\mu_{3} \operatorname{sech}^{2}\left(\alpha\left(\mu_{0}+D\right)\right) & u_{3}^{\prime}-i \mu_{2} u_{1}-u_{3}\left(i \mu_{0}+\partial_{x}\right)+i \mu_{3} c_{0}
\end{array}\right) .
$$

Evaluating (A25) at $\eta_{j}, u_{j}$, and $\mathbf{w}_{\mathbf{j}-\mathbf{1}}$ for $j \in\{1,2,3\}$, one finds

$$
\left(\mathcal{L}_{0, \mu_{0}}-\lambda_{0}\right) \mathbf{w}_{3}=\sum_{\substack{j=n-3 \\ j \neq n-2}}^{m+3} \mathbf{T}_{3, j} e^{i j x},
$$

where $\mathbf{T}_{3, \mathbf{n}-2}=\mathbf{0}$.

The solvability conditions for (A27) are

$$
\begin{array}{r}
2\left(\lambda_{3}+i \mu_{3} c_{g_{1}}\left(\mu_{0}+n\right)\right)+i \gamma_{0} \mathcal{S}_{3, n}=0 \\
2 \gamma_{0}\left(\lambda_{3}+i \mu_{3} c_{g_{-1}}\left(\mu_{0}+m\right)\right)+i \mathcal{S}_{3, m}+i \gamma_{1} \mathcal{T}_{3, m}=0
\end{array}
$$


where $\mathcal{S}_{3, j}$ and $\mathcal{T}_{3, m}$ have no dependence on $\gamma_{0}, \gamma_{1}, \mu_{3}$, or $\lambda_{3}$; see the file in Data Availability Statement. Using (A22a) and (A22b) from the previous order as well as (24), one can show that $\mathcal{T}_{3, m} \equiv 0$. In addition, similar to (47) for the $p=2$ isola, we have

$$
\mathcal{S}_{3, n} \mathcal{S}_{3, m}=-\frac{\mathcal{S}_{3}^{2}}{\omega_{\alpha}\left(\mu_{0}+m\right) \omega_{\alpha}\left(\mu_{0}+n\right)},
$$

where $\mathcal{S}_{3}$ is given in the file in Data Availability Statement. As a result, (A28a) and (A28b) form a nonlinear system for $\lambda_{3}$ and $\gamma_{0}$.

\section{References}

1. Whitham, G. Variational methods and applications to water waves. Proc. R. Soc. A 1967, 299, 6-25.

2. Whitham, G. Non-linear dispersion of water waves. J. Fluid Mech. 1967, 27, 399-412. [CrossRef]

3. Deconinck, B.; Trichtchenko, O. High-frequency instabilities of small-amplitude of Hamiltonian PDE's. Discret. Contin. Dyn. Syst. A 2017, 37, 1323-1358.

4. Hur, V.; Pandey, A. Modulational instability in a full-dispersion shallow water model. Stud. Appl. Math. 2019, 142, 3-47. [CrossRef]

5. Hur, V.; Tao, L. Wave breaking in a shallow water model. SIAM J. Math. Anal. 2019, 50, 354-380. [CrossRef]

6. Nekrasov, A. On steady waves. Izv. Ivanovo-Voznesensk. Politekhn. In-ta 1921, 3, 52-65.

7. Stokes, G. On the theory of oscillatory waves. Trans. Camb. Philos. Soc. 1847, 8, 441-455.

8. Struik, D. Determination rigoureuse des ondes irrotationnelles periodiques dans un canal à profondeur finie. Math. Ann. 1926, 95, 595-634. [CrossRef]

9. Chicone, C. Ordinary Differential Equations with Applications, 2nd ed.; Springer: New York, NY, USA, 2006.

10. Kapitula, T.; Promislow, K. Spectral and Dynamical Stability of Nonlinear Waves, 1st ed.; Springer: New York, NY, USA, 2013.

11. Reed, M.; Simon, B. Methods of Modern Mathematical Physics, IV. Analysis of Operators, 2nd ed.; Academic Press-Harcourt Brace Jovanovich Publishers: New York, NY, USA, 1978.

12. Deconinck, B.; Kutz, J. Computing spectra of linear operators using the Floque-Fourier-Hill method. J. Comput. Phys. 2006, 219, 296-321. [CrossRef]

13. Haragus, M.; Kapitula, T. On the spectra of periodic waves for infinite-dimensional Hamiltonian systems. Phys. D Nonlinear Phenom. 2008, 237, 2649-2671.

14. Johnson, M.; Zumbrun, K.; Bronski, J. On the modulation equations and stability of periodic generalized Korteweg-de Vries waves via Bloch decompositions. Phys. D Nonlinear Phenom. 2010, 239, 2057-2065. [CrossRef]

15. Benjamin, T. Instability of periodic wave trains in nonlinear dispersive systems. Proc. R. Soc. Lond. A 1967, 299, 59-79.

16. Benjamin, T.; Feir, J. The disintegration of wave trains on deep water. part i. theory. J. Fluid Mech. 1967, 27, 417-430. [CrossRef]

17. Bridges, T.; Mielke, A. A proof of the Benjamin-Feir instability. Arch. Ration. Mech. Anal. 1995, 133, 145-198. [CrossRef]

18. Claassen, K.; Johnson, M. Numerical bifurcation and spectral stability of wavetrains in bidirectional Whitham models. Stud. Appl. Math. 2018, 141, 205-246. [CrossRef]

19. Deconinck, B.; Oliveras, K. The instability of periodic surface gravity waves. J. Fluid Mech. 2011, 675, 141-167. [CrossRef]

20. Trichtchenko, O.; Deconinck, B.; Wilkening, J. The instability of Wilton ripples. Wave Motion 2016, 66, 147-155. [CrossRef]

21. Creedon, R.; Deconinck, B.; Trichtchenko, O. High-frequency instabilities of the Kawahara equation: A perturbative approach. arXiv 2021, arXiv:2101.06601.

22. Akers, B. Modulational instabilities of periodic traveling waves in deep water. Phys. D Nonlinear Phenom. 2015, 300, 26-33. [CrossRef]

23. Bronski, J.; Hur, V.; Johnson, M. Modulational instability in equation of KdV-type. In New Approaches to Nonlinear Waves. Lecture Notes in Physics; Tobisch, E., Ed.; Springer: Cham, Switzerland, 2016; pp. 83-133.

24. Akers, B.; Nicholls, D. The spectrum of finite depth water waves. Eur. J. Mech. B/Fluids 2014, 46, 181-189. [CrossRef]

25. Krein, M. On the application of an algebraic proposition in the theory of matrices of monodromy. Uspekhi Mat. Nauk. 1951, 6, 171-177.

26. MacKay, R.; Saffman, P. Stability of water waves. Proc. R. Soc. A 1986, 406, 115-125.

27. Kato, T. Perturbation Theory for Linear Operators, 1st ed.; Springer: Berlin, Germany, 1966. 